\title{
Bubble Glow at Hydrothermal Vents as the PeTa Radiation
}

\author{
Vitali A. Tatartchenko \\ CNRS, Aix-Marceille University, Marseille, France \\ Email: vitali.tatartchenko@orange.fr
}

How to cite this paper: Tatartchenko, V.A. (2019) Bubble Glow at Hydrothermal Vents as the PeTa Radiation. Optics and Photonics Journal, 9, 189-217. https://doi.org/10.4236/opj.2019.911017

Received: October 25, 2019

Accepted: November 23, 2019

Published: November 26, 2019

Copyright (C 2019 by author(s) and Scientific Research Publishing Inc. This work is licensed under the Creative Commons Attribution International License (CC BY 4.0).

http://creativecommons.org/licenses/by/4.0/

\begin{abstract}
The paper presents a physical model of a natural phenomenon, the glow of bubbles at hydrothermal vents formed during underwater volcanic activity. The basis of the model is characteristic non-equilibrium radiation under first order phase transitions that since 2010 has been referred to as the PeTa (Perelman-Tatartchenko) effect. This is the fourth paper in a series developing the model for similar physical phenomena: cavitational luminescence $(C L)$, multi-bubble sonoluminescence ( $M B S L)$, single-bubble sonoluminescence $(S B S L)$ and laser-induced bubble luminescence (LIBL). The previous three papers were published during 2017-2018 in this Journal. In the third one we have shown that above mentioned physical effects can be generalized as a phenomenon that we have titled "Vapour bubble luminescence" (VBL). VBL is very clearly represented in a non-equilibrium phase diagram. The essence of $V B L$ is as follows: when there is a local decrease in pressure and/or an increase of temperature in a tiny volume of a liquid occurs, one or several bubbles filled with vapour will appear. Subsequently a very rapid pressure increase and/or temperature decrease in the same volume of liquid leads to supersaturation of the vapour inside the bubble. Upon reaching critical vapor density, instantaneous vapour condensation and emission of the phase transition energy that is accompanied by a flash (this is the PeTa effect) results in a sharp pressure decrease and the bubble collapses due to the pressure drop. This process is accompanied by a shock wave in the liquid. A similar effect occurs if bubbles filled with hot steam, for example from a cappuccino machine, are injected into a relatively large volume of cold water. The $V B L$ model explains all experimental data concerning $C L / M B S L / S B S L / L I B L$ and the relatively new natural phenomenon, the glow of bubbles at hydrothermal vents. Several model experiments demonstrate the PeTa effect under similar conditions. Additionally, we define the PeTa effect in all its manifestations on a non-equilibrium phase diagram. This clarifies which niches can contain $V B L$ processes. We also demonstrate the window of transparency (WT) for
\end{abstract}


the $P e T a$ radiation during crystallization of a supercooled tellurium melt and propose the design of a cavity-free pulsed laser on the basis of similar crystallization processes.

\section{Keywords}

Bubble Glow, Hydrothermal Vents, Perel'man-Tatartchenko (PeTa) Effect, Cavitational Luminescence $(C L)$, Sonoluminescence $(S L)$, Vapour Bubble Luminescence $(V B L)$

\section{Introduction}

This is the fourth paper in a series that presents steps in the development of a Perelman-Tatartchenko ( $P e T a$ ) model for similar physical phenomena: cavitational luminescence $(C L)$, multi-bubble sonoluminescence $(M B S L)$, single-bubble sonoluminescence $(S B S L)$ and laser-induced bubble luminescence (LIBL). The previous three papers were published during 2017-2018 in this Journal [1] [2] [3]. The basis of the PeTa model is characteristic non-equilibrium radiation under first order phase transitions that since 2010 has been referred to as the PeTa effect [4]. It should be noted that we have previously proposed that the PeTa effect could be a source of MBSL [5] [6] [7]. However, it was done without detailed analyses and concerned only infrared radiation in $M B S L$ [8]. We later developed a detailed first iteration of the model [1]. The model explained the main experimental results of $C L / M B S L / S B S L$. The model was then improved [2], and we showed that it is able to interpret additional features of the aforementioned phenomena. This is especially true for $L I B L$, in which vapour bubbles form in the liquid due to a short laser pulse. Interesting peculiarities of the $L I B L$ spectrum in water were explained. In the third paper [3], we interpreted the spectra of the $L I B L$ in cryogenic liquids. In addition, we continued to improve our model. We have shown that the similar physical effects under consideration $(C L / M B S L / S B S L / L I B L)$ can be generalized as a phenomenon that we have titled "Vapour bubble luminescence" $(V B L) . V B L$ is very clearly represented in a non-equilibrium phase diagram (Figure 3 from [3]). The essence of $V B L$ is as follows: when there is a local decrease in pressure and/or an increase of temperature in a tiny volume of the liquid occurs, one or several bubbles filled with vapour will appear. Subsequently a very rapid pressure increase and/or temperature decrease in the same volume of liquid leads to supersaturation of the vapour inside the bubble. Upon reaching critical vapor density, instantaneous vapour condensation and emission of the phase transition energy that is accompanied by a flash (this is the PeTa effect) results in a sharp pressure decrease and the bubble collapses due to the pressure drop. This process is accompanied by a shock wave in the liquid. A similar effect occurs if bubbles filled with hot steam, for example from a cappuccino machine, are injected into a relatively large volume of cold water. The $V B L$ model explains all experimental data concerning 
$C L / M B S L / S B S L / L I B L$ phenomena [1] [2] [3]. We have also proposed the design of a cavity-free pulsed laser on the basis of $V B L$ luminescence [3]. Indeed, the vapor is in the supercooled state inside the bubble before the flash. All atoms/molecules and clusters are united in a single system by the electromagnetic field of interaction. During the flash, the excitation energy of all particles of the cloud is instantaneously released. We described in detail all these processes in the paper [2] based on the analogy with super-radiation. It is obvious that in many respects, our system is similar to a cavity-free pulsed laser. The difference lies in the fact that the radiation is nonmonochromatic because the system contains several types of excited particles that emit simultaneously, i.e. it can be interpreted as the simultaneous emission of several cavity-free pulsed lasers. If necessary, these emissions can be separated.

In this paper, we first analyse a relatively new natural phenomenon, the glow of bubbles formed during underwater volcanic activity, and several additional experiments that demonstrate the PeTa effect under similar conditions. Second, we define the $P e T a$ effect in all its manifestations using a non-equilibrium phase diagram. This clarifies which niches can contain $V B L$ processes. Third, we analyse other physical experiments and natural effects that can be described by the PeTa model. This primarily concerns the analysis of the PeTa radiation during the crystallization of supercooled tellurium melt. We also propose the design of cavity-free pulsed lasers on the basis of similar crystallization processes.

\section{Bubble Luminescence during Hydrothermal Activity}

\subsection{Field Observations}

Bubble luminescence at great ocean depths was observed as a result of the scientific collaboration of geophysicists and biologists. Plate tectonic theory had predicted the existence of hydrothermal vents, which are deep-sea hot springs that form when cold sea water seeps into cracks on the ocean floor, heats up, and rises. The existence of hydrothermal vents was confirmed in 1977, as part of the Galápagos Hydrothermal Expedition [9], with the help of the research submersible Alvin. Since 1977, more than 500 vent sites have been discovered along the mid-ocean ridges that separate some of Earth's tectonic plates.

In 1989, researchers observed that the Rimicaris exoculata deep-sea shrimp living in vent areas have photoreceptors similar to those of humans and are sensitive to daylight [10]. The presence of these photoreceptors indicates that sources of this kind of light must be near the shrimp. Daylight itself does not penetrate to these depths, meaning additional sources of light must be present. Cherenkov's radiation was an initial candidate, but the intensity of Cherenkov's radiation is too weak to be registered by the photoreceptors. Instead, the sources were the hightemperature hydrothermal vents themselves [11]. Planck radiation was ruled out because the maximum temperature of the vents did not exceed $400^{\circ} \mathrm{C}$, and the vents could only emit in the infrared range. Currently, the nature of glow from high-temperature hydrothermal vents glow has not been explained. In the follow- 
ing sections we will explain the nature of these light sources in the deep ocean.

\subsection{Hydrothermal Vent Structures}

With respect to [12], three main high-temperature vent structures exist: Black smoker chimneys, beehives, and flange pools.

Hydrothermal vents often form features called black smokers: black, chimney-like structures that emit superheated mineral-rich fluids (Figure 1). Black smokers form in fields on a scale of hundreds of meters wide when superheated water from inside the crust vents at the ocean floor (fluid temperatures may reach above $400^{\circ} \mathrm{C}$ ). The fluid is rich in dissolved minerals derived from the crust, most notably metallic sulfides. When the fluid comes in contact with cold

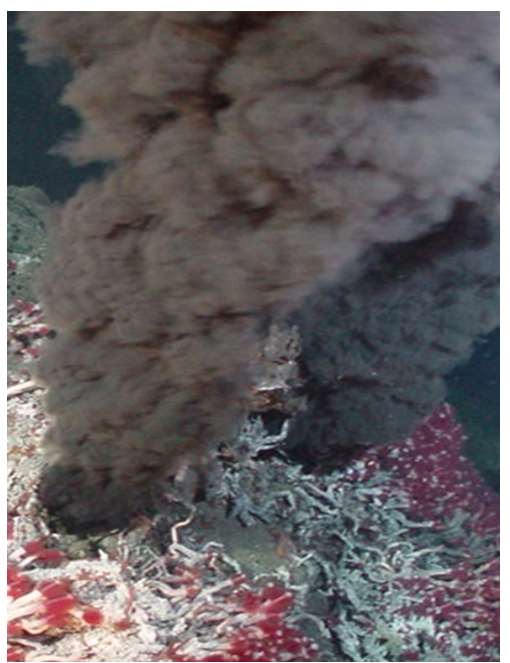

Figure 1. From https://oceanservice.noaa.gov/facts/vents.html: View of Black smoker and increased biological activity around it.

(a)

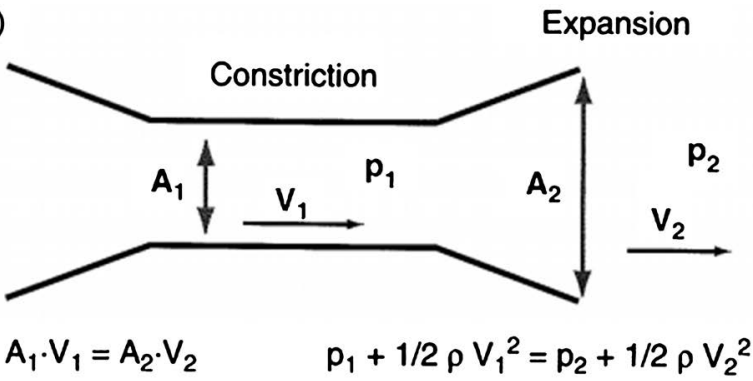

(b)

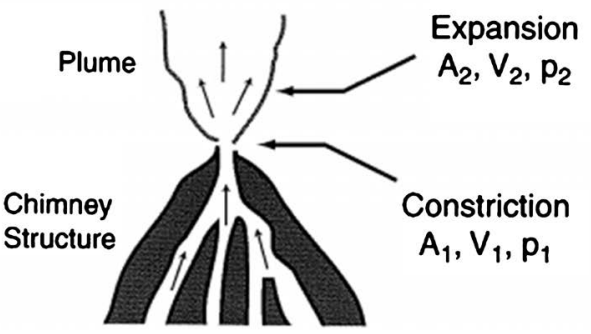

Figure 2. From [13]: (a) analogy with Venturi tube; (b) Scheme of Black smoker. 
bottom water $\left(\sim 2^{\circ} \mathrm{C}\right)$ minerals precipitate, forming a chimney structure around the vent. Figure 2(a) \& Figure 2(b) shows a flow pattern in a black smoker (Figure 2(b)) and an analogous flow scenario in a Venturi tube (Figure 2(a)). The flow rate of the fluid $(v)$, the pressure inside the fluid $(P)$ and the cross sectional area $(A)$ are interconnected by the laws of mass conservation (left formula) and energy conservation (right formula). When the fluid exits the orifice of a black smoker, its pressure drops sharply, which leads to the formation of numerous bubbles. We will carry out further analyses of the processes associated with black smoker chimney activity below.

The second type of high temperature vent structure is a beehive (Figure 3). These consist of porous mineral materials. The structure itself is primarily a matrix of anhydrite $\left(\mathrm{CaSO}_{4}\right)$, with lesser amounts of pyrite, chalcopyrite, sphalerite, and wurtzite. In these structures, the venting of rather clear fluid, and the formation of bubbles is possible only from a system of channels that end at the surface of the structure, visible as holes (Figure 3(c))

In addition to black smoker chimneys and beehives, flange pools (high-temperature fluids trapped beneath mineralised sulfide-sulfate-silica flanges) are also observed at many vent sites. Figure 4(a) is a diagram of the release of superheated fluid from this system. The fluid has two ways to exit: the lateral and central channels. Only the lateral channel, which is visible in Figure 4(b), works in this scenario. Fluid movement here is laminar and the surface (Figure $4(c)$ ) is

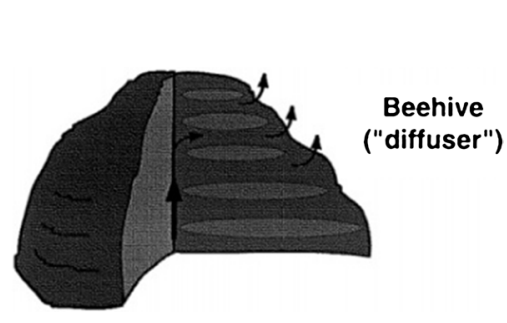

(a)

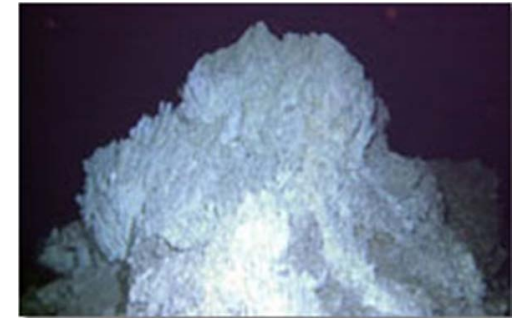

(b)

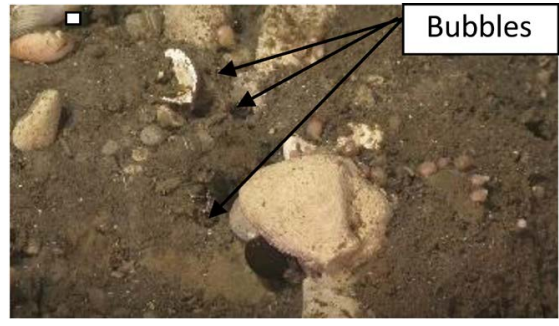

(c)

Figure 3. Beehives; (a) scheme (from [13]); (b) view; (c) bubbls appeared from holes.

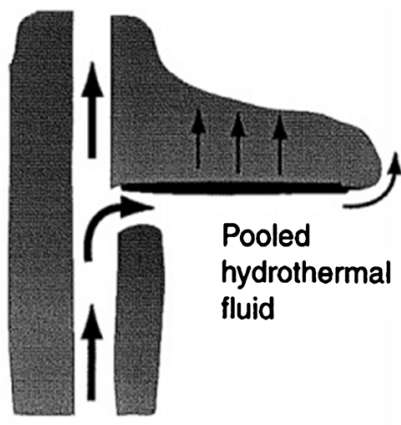

Flange Pool

(a)

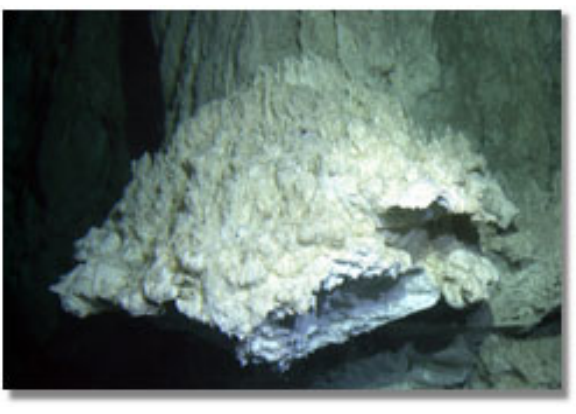

(b)

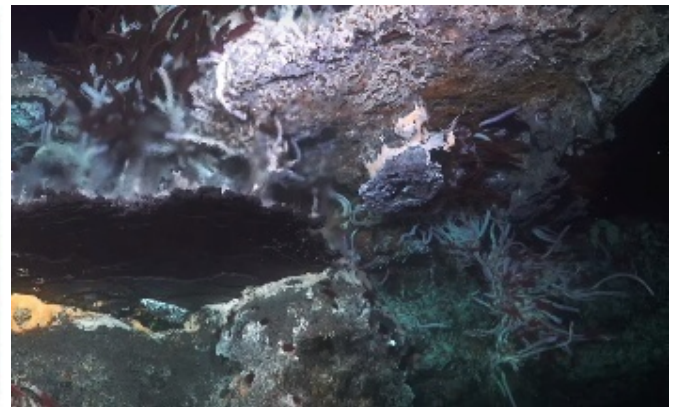

(c)

Figure 4. From [13]: Scheme of flange pool. (b) and (c) (from https://www.nsf.gov/od/lpa/news/press/00/pr0093.htm), (a) carbonate ledges or flanges that extend outwardly from the trunk of a 50 meters chimney in the hydrothermal vent field. The flanges trap pools of $70^{\circ} \mathrm{C}$ fluids. The nutrient-rich fluids support dense microbial communities; (b) lateral view, (c) top view. 
calm, explaining the name of these formations. The pools are often visible as a mirror effect (Figure 5(a)). This fluid behaviour is surprising, and so far no one has explained a physical mechanism for this phenomenon. However, we can address this based on our previous research.

\subsection{Physical Nature of Flange Pool Features}

Since the 1970s, we have been investigating similar phenomena and have discovered their physical mechanisms [14] [15]. This helps understanding of the flange pool features. Along with the unusual outlet of venting fluids from the flange pools described previously, there are two very interesting features. First, a large part of the vertical structure is a cylinder (Figure 4(a)). Second, fluid pooled beneath these flanges is stratified, forming a sharp interface with the ambient $\left(\sim 2^{\circ} \mathrm{C}\right)$ bottom sea water, across which minimal mixing occurs. This results in the mirror surface effect that is observed (Figure 5(a)).

The first series of experiments [14] was as follows. In a test tube containing a room temperature solution of some salt, a gradient of solute concentrations along the axis of tube was created. The density of the solution decreased from the bottom up. The tube was immersed in a vessel containing water at a temperature of $\sim 90^{\circ} \mathrm{C}$. After several seconds in a parallel light beam, the formation of layers with different optical refractive index values was detected in the test tube (Figure 5(b)). A similar effect occurred when the preheated tube was suddenly cooled. The abrupt change in the refractive index was due to a sharp change in the density of the solution in the adjacent layers, because after a long time the clear boundary between the layers disappeared due to diffusion. The layers that formed in the region with the highest concentration gradient changed over time.

The second series of experiments [15] confirmed the proposed mechanism and showed the existence of vortex cells. In Figure 5(c), a heater visible at the

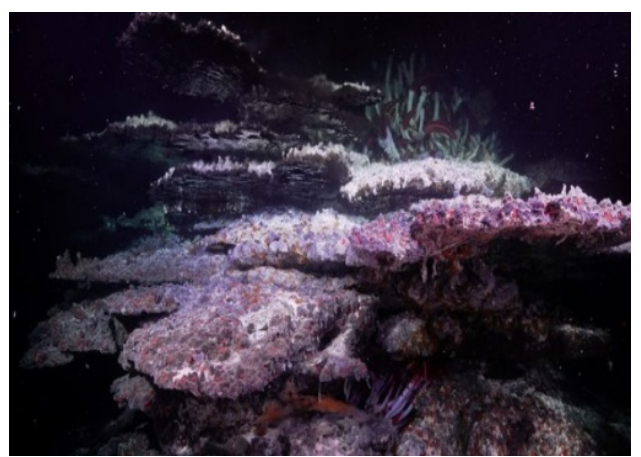

(a)

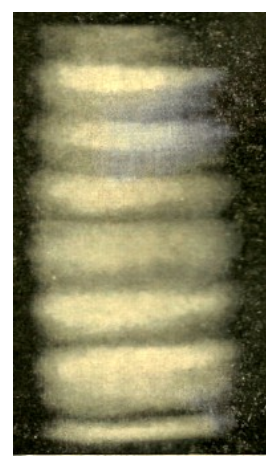

(b)

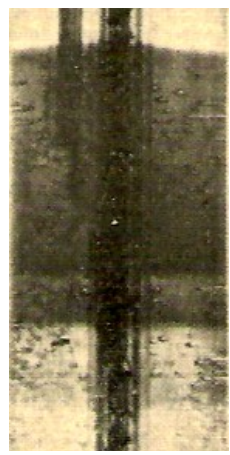

(c)

Figure 5. (a) From

[https://www.geek.com/news/scientists-find-mesmerizing-mirror-like-pools-in-the-pacifi c-ocean-1781759/]: Mirror flange pool; (b) From [14]: Density strata in a fluid with a vertical density gradient and a horizontal temperature gradient; (c) From [15]: Vortex cells in a liquid with a horizontal temperature gradient and a vertical salt concentration gradient, detected by staining. In the center of the vessel is visible heater, on the left-a tube for paint injection. 
centre of the vessel created a horizontal temperature gradient in a liquid with a vertical salt concentration gradient. The resulting cells were tinted with dye. A sharp boundary between the cells was observed, indicating that there is little to no fluid exchange between the cells, and that the vortex flows inside the cells to evenly mix the paint. The trajectories of the motion of bubbles (Figure 5(c)) indicate the presence of intense convection in each cell from the axis to the periphery, and vice versa.

If the experiment is carried out according to the schematic in Figure 5(c), but with a homogeneous solution, the paint injection uniformly stains of all the liquid in the vessel. Thus, there is one large convective cell. The heated liquid rises upward at the centre of the test tube and descends downwards at its walls. This means that at the upper border of the cell, the liquid travels from the centre to the periphery, and at the lower border, vice versa. If instead there is a concentration gradient of dissolved salt in the test tube, with the density of the solution decreasing from bottom to top, for convection to occur in the centre of the tube, the decrease in density in the lower layer due to heating should exceed its increase due to its concentration. Along the axis of the tube, where this condition is satisfied, the solution begins to move upwards. But in some places, the density of the colder, less saturated solution is equal to the density of the hotter, but more saturated solution. In this case, instability occurs, and to maintain the continuity of flow, the solution begins to move horizontally. The whole solution is divided into such cells. The number of cells is equal to the number of points along the axis of the tube where instability occurrs. Inside each cell, the concentration is averaged, and then convection is implemented in the usual way. The cellular system is fairly stable, because the change in concentration across the cell boundary can occur only due to diffusion, and this is a very slow process. It should be noted that for aqueous solutions of most salts, a 3\% change in concentration is equivalent to a $50^{\circ} \mathrm{C}$ temperature change.

A similar situation is likely in the cylindrical part of the flange pool. The cellular convective structure provides great resistance to fluid movement through the central channel. Therefore, the venting occurs instead through the lateral holes.

\subsection{Vent Glow and Spectral Imaging}

In 1988, work at the Endeavor vent field on the Juan de Fuca range obtained the first image of a light source from deep-water hydrothermal vents using a chargecoupled device $(C C D)$ [11] [13] [16]. This image (Figure 6(a)) clearly showed the light radiating from the vent and decreasing in intensity with increased distance above the orifice. To obtain the spectrum, a simple "Optical properties of an underwater sensor" (OPUS) photometer of the first generation was built [13] [17]. Measurements using OPUS were carried out on high-temperature hydrothermal vents on the Mid-Atlantic Ridge and the East Pacific Rise. Radiation in the range of $650-750 \mathrm{~nm}$ was observed, which is $\sim 20$ times larger than the expected Planck radiation (Figure 6(b)). In addition, temporary changes were recorded (Figure $6(\mathrm{c})$ ) that could not be explained by thermal radiation from a 


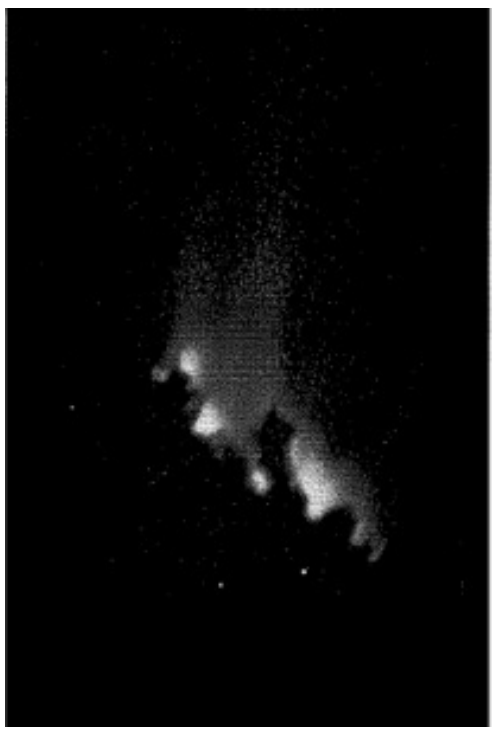

(a)

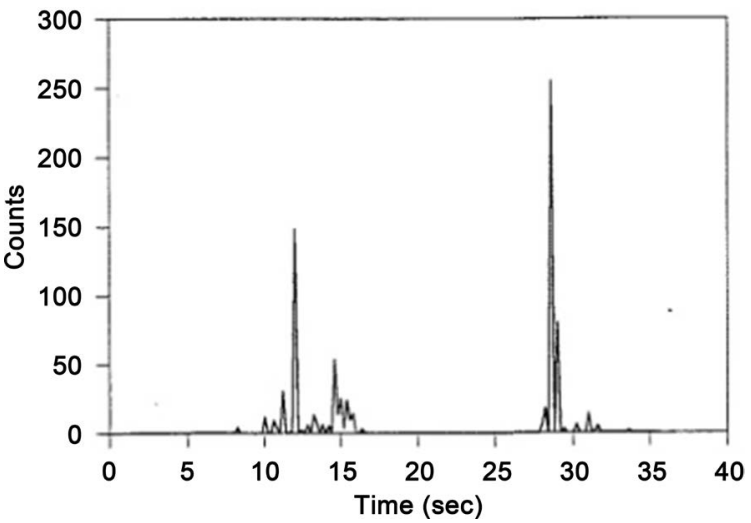

(c)

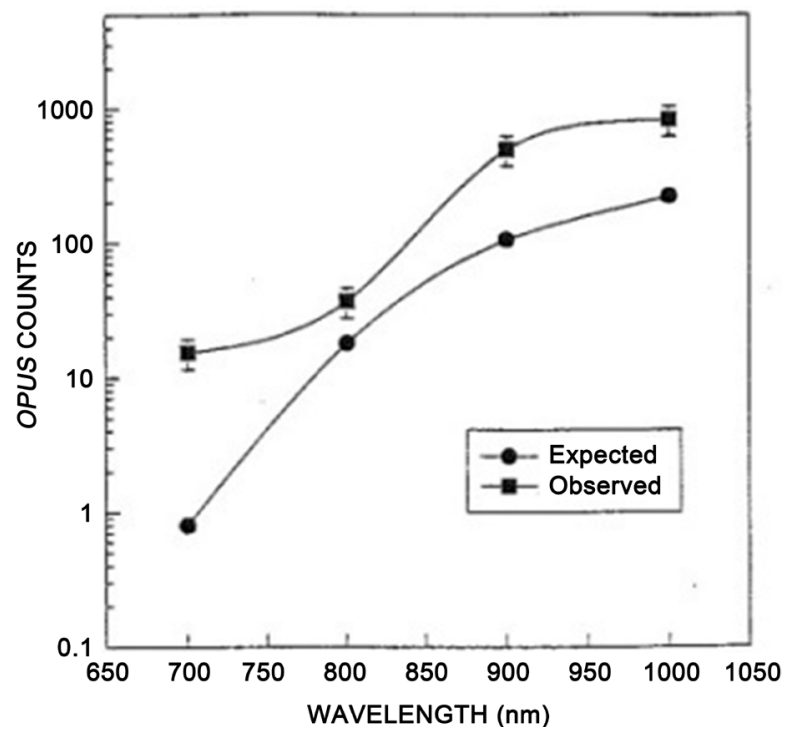

(b)

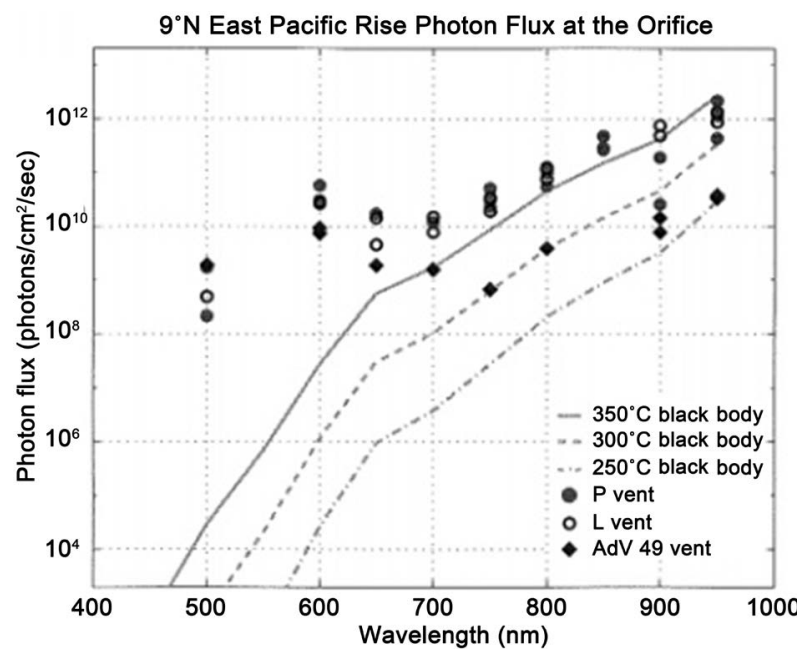

(d)

Figure 6. (a) From [16]: First image of vent glow taken with a $C C D$ camera at a vent on the Endeavour Segment of the Juan de Fuca Ridge during an Alvin dive cruise in 1988; (b) From [17]: Observed OPUS counts at a vent compared to expected counts from a $350^{\circ} \mathrm{C}$ black body source; plotted points have a bandwidth of $100 \mathrm{~nm}$ and $25 \%$ error bars; (c) From [17]: Time series of OPUS counts in the $800 \mathrm{~nm}$ channel observed $10 \mathrm{~cm}$ above a vent; (d) From [19]: OPUS data from three vents compared to radiation from $250^{\circ} \mathrm{C}, 300^{\circ} \mathrm{C}$ and $350^{\circ} \mathrm{C}$ black body sources. $\mathrm{P}$ vent as well as $\mathrm{L}$ vent has a temperature of $375^{\circ} \mathrm{C}, \mathrm{AdV} 4$ - 9 vent has a temperature of $260^{\circ} \mathrm{C}$.

constant temperature source. This indicated the need for recording the visible part of the spectrum.

In the second generation of the OPUS device [18], by doubling the number of diodes, the measurement range was expanded to the visible spectrum. This allowed researchers to show that vents emit visible light Figure 6(d) [13] [19].

\subsection{The ALISS Device Used to Measure Intensity and Spectra of Underwater Radiation}

A more sophisticated Ambient Light Imaging and Spectral System (ALISS)was 
built to further address the visible spectrum [13] [18] [19] [20] [21] [22]. It was ALISS that made it possible to obtain complete data on the spectra of various vents and to associate the glow with the presence of vapor bubbles.

ALISS uses a CCD camera, but unlike the OPUS instruments, ALISS can obtain both spectral and spatial information for simultaneously imaging vents in nine wavelength bands. Taking a number of short-exposure images provides temporal information, but a long exposure and image processing techniques allow for the measurement of very low light levels.

In the ALISS instrument, the $1024 \times 1024$ pixel $C C D$ chip is divided into nine $\sim 300 \times 300$ pixel tiles by a set of baffles and lenses. Each lens is covered with an individual optical bandpass filter. ALISS contains two filter arrays of nine filters each which span the $400-1000 \mathrm{~nm}$ range of the spectrum (i.e. the visible and near infrared). Filter array \#1 contains nominally $100 \mathrm{~nm}$ bandwidth filters centred at $450,550,599,652,705,753,792,870$, and $947 \mathrm{~nm}$. Filter array \#2 contains two $100 \mathrm{~nm}$ bandwidth filters centred at 492 and $902 \mathrm{~nm}$, six nominally 50 $\mathrm{nm}$ bandwidth filters centred at 475,577,676,780, 878, and $980 \mathrm{~nm}$, and a 10 $\mathrm{nm}$ bandwidth filter at $589 \mathrm{~nm}$. Figure 7 shows that the device has an almost uniform effective aperture in the visible and near infrared regions of the spectrum. The effective aperture $\left(\mathrm{cm}^{2}\right)$ includes the entrance pupil of the lens, transmission of the filter, quantum efficiency of the $C C D$ chip, and any other internal losses in the optical assembly. The effective aperture determined in air was divided by 1.77 (the square of the index of refraction of water) to account for the smaller entrance pupil when used in water rather than air.

\subsection{Experimental Implementation}

The ALISS camera was deployed at two vent sites in the Pacific: the Venture Hydrothermal Field $\left(9^{\circ} \mathrm{N}\right.$ EPR) and the Main Endeavour Field on the Juan de Fuca Ridge. It was located $50 \mathrm{~cm}$ from a black smoker chimney and a flange pool, as shown in Figure 8. For each filter matrix, several 5-minute images were taken. In some cases, series of 30-second images were taken to analyze time-variable light. During exposures, all possible light sources were covered or turned off. This

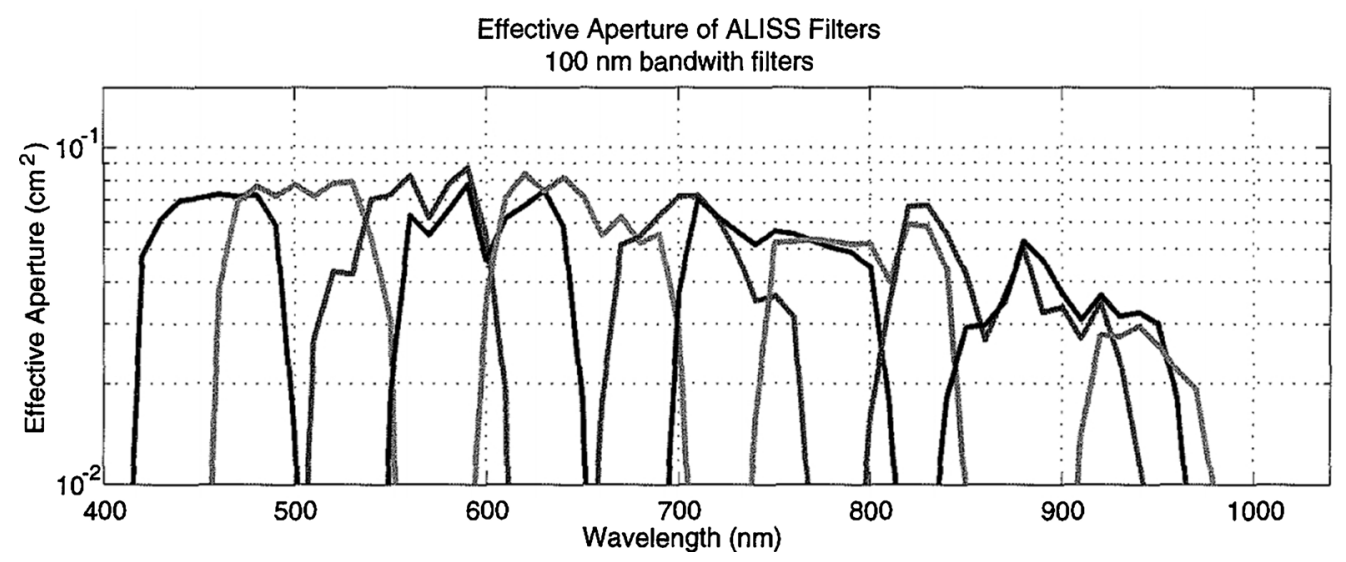

Figure 7. From [13]; effective apperture vs wavelength for the device ALISS. 
made it possible to detect and analyze even very low levels of light (of the order of $10^{3}$ photons $\cdot \mathrm{cm}^{-2} \cdot \mathrm{s}^{-1}$ per steradian at a distance of $50 \mathrm{~cm}$ in water), and the emitting areas were localized.

\subsection{Measurement Results}

Figures show typical results of the emission spectra respectively from: a flange pool (Figure 9); a beehive (Figure 10); and a black smoker chimney (Figure $11)$.

\subsubsection{Flange Pools}

In Figure 9, data from the $100 \mathrm{~nm}$ bandwidth filters are compared to the calculated flux for a theoretical black body with a temperature of $332^{\circ} \mathrm{C} \pm 5^{\circ} \mathrm{C}$ and

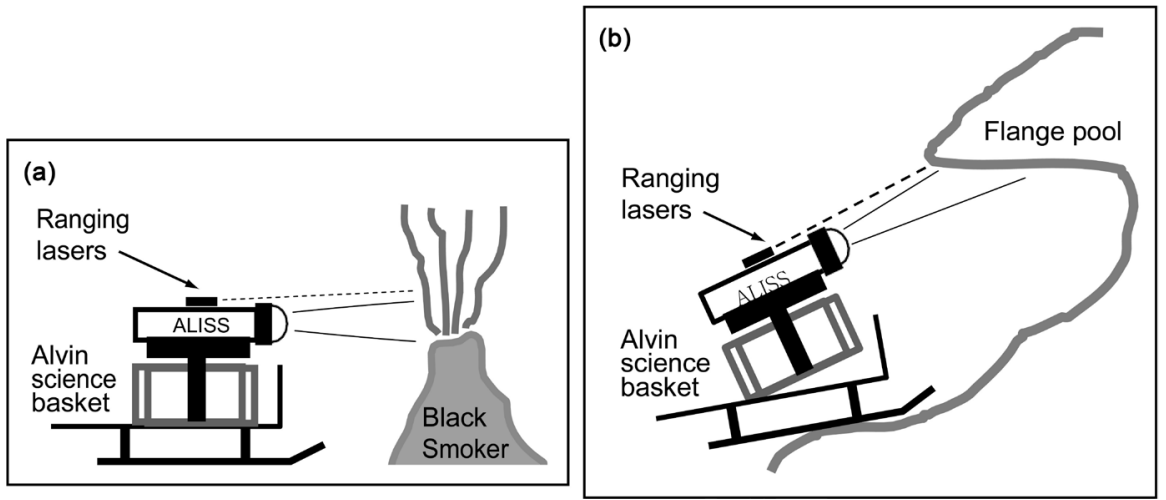

Figure 8. From [13] schematic showing how ALISS is positioned at (a) black smokers and (b) flanges pools. Ranging lasers cross at $50 \mathrm{~cm}$ from the optical window (the focal point of the ALISS camera).

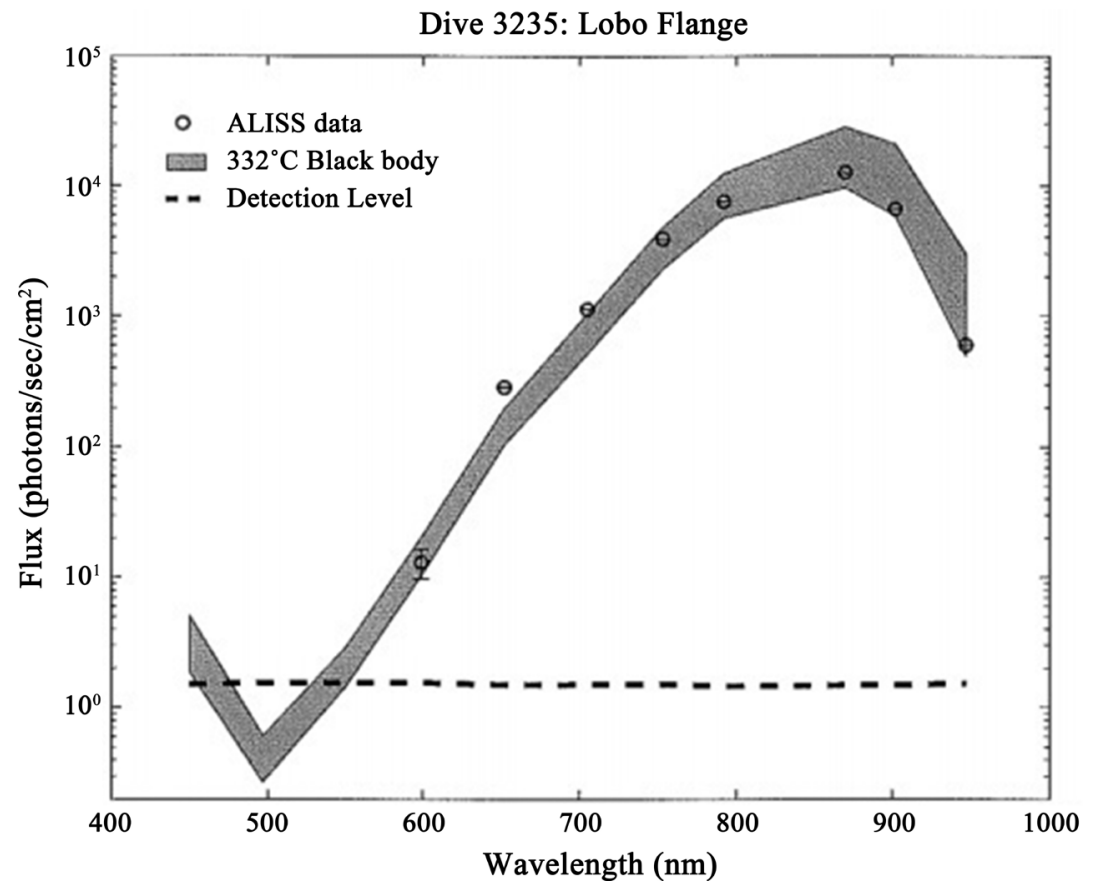

Figure 9. From [13]: Spectral data from Lobo flange. 


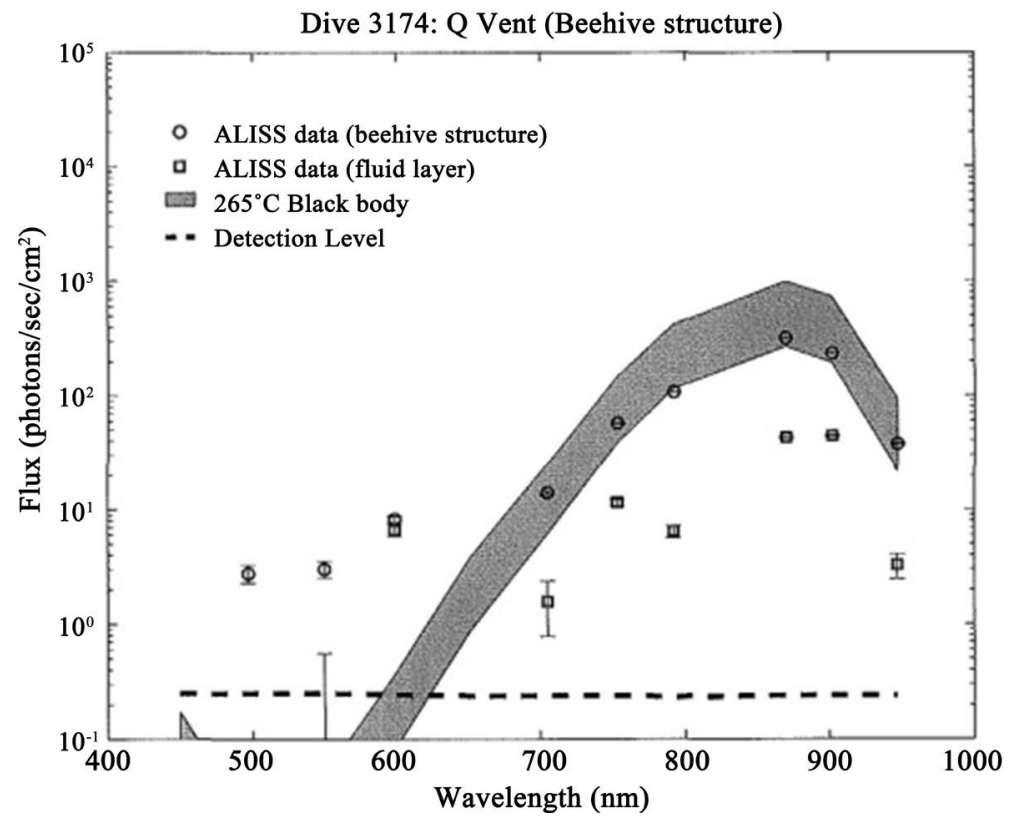

Figure 10. From [13]: Spectral data from the beehive at Q Vent (dive 3174).

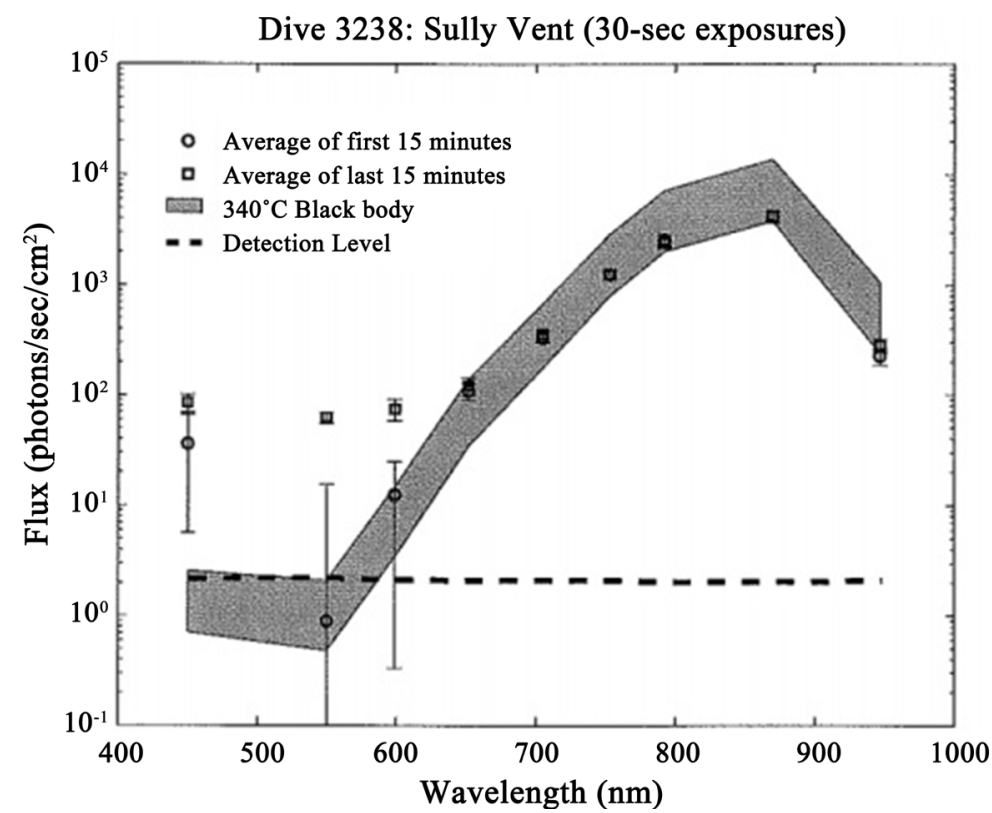

Figure 11. From [13]: Spectral data from a black smoker of Sully Vent (dive 3238).

emissivity of $0.9 \pm 0.1$, at a distance of $50 \pm 10 \mathrm{~cm}$ as observed through the ALISS camera. The drop-off in flux above $870 \mathrm{~nm}$ is due to high attenuation of light in sea water at those wavelengths. The higher flux predicted at $450 \mathrm{~nm}$ for a theoretical black body is due to a red leak in that particular filter. The ALISS data from the 450, 500, and $550 \mathrm{~nm}$ filters were below detection level. From Figure 8(b) it is obvious that during these measurements, ALISS was positioned in such a way as to record the radiation of both the flange and side flows of the venting fluid. Experimental data recorded the presence of radiation only from a black body heated to a temperature of $332^{\circ} \mathrm{C} \pm 5^{\circ} \mathrm{C}$. 


\subsubsection{Beehives}

In Figure 10, data from several experiments from the $100 \mathrm{~nm}$ bandwidth filters are shown. The data are compared to the calculated flux for a theoretical black body with a temperature of $265^{\circ} \mathrm{C} \pm 10^{\circ} \mathrm{C}$ and emissivity of $0.9 \pm 0.1$, at a distance of $50 \pm 5 \mathrm{~cm}$ from the ALISS camera. The drop-off in flux above $870 \mathrm{~nm}$ is due to the high attenuation of light in seawater at those wavelengths. The presence of weak radiation is observed in the visible region of the spectrum.

\subsubsection{Black Smoker Chimneys}

In Figure 11, the spectra from sixty 30 -second exposure images are compared to the calculated flux for a theoretical black body with a temperature of $340^{\circ} \mathrm{C} \pm$ $10^{\circ} \mathrm{C}$ and emissivity of $0.3 \pm 0.1$, at a distance of $50 \pm 5 \mathrm{~cm}$ from the ALISS camera. The presence of radiation in the visible region of the spectrum is obvious. The intensity of this radiation in black smoker chimneys is $\sim 2$ orders of magnitude higher than in beehives.

\subsection{Assumptions about the Nature of Radiation in the Visible Region of the Spectrum}

The appearance of radiation in the visible region of the spectrum has been associated with the formation of bubbles [13] [18] [19] [20] [21] [22]. Indeed, in the study of flange pools, recorded radiation was only emitted by heated rocks and the laminar fluid flows. There were no bubbles generated. The recorded radiation is fully consistent with Planck radiation at a temperature of $\sim 300^{\circ} \mathrm{C}$. In the study of beehives and black smoker chimneys, along with the Planck radiation, radiation was registered in the visible region of the spectrum. In these cases, the radiation power in beehives was $\sim 2$ orders of magnitude lower than in the chimneys. The number of bubbles correlated with the radiation power. In the case of the beehives, rare bubbles emerge from the porous rock. But in black smoker chimneys, hot water vents from the orifice (like a Venturi tube) and the pressure drops, causing numerous bubbles to form. Previous studies have suggested that this glow is similar to $C L$ and $S L$, the nature of which, however, is still unknown to the authors [13] [18] [19] [20] [21] [22]. To verify this assumption, very reasonable modeling experiments were carried out.

\section{Modeling Experiments}

\subsection{Artificial Bubble Luminescence}

Chakravarty and Walton, in order to clarify the mechanism of the glow of vent bubbles, carried out modeling experiments [23]. They reported observing light from a process associated with macroscopic vapour bubbles when superheated steam bubbles condensed in water. The steam was produced in a cappuccino machine, by attaching the frothing attachment to a glass tube that ended several centimeters below the water surface in a rectangular tank. Steam bubbles were introduced through a $1 \mathrm{~mm}$ bore glass nozzle at a rate of $\sim 1000$ bubbles/s into water at room temperature. High-speed video recordings made under normal 
lab lighting confirmed that the collapsing bubbles remained separate from one another. The luminescence was observed using a high-gain image intensifier (IIT) microscope system ( $4 \times$ magnification) with a spectral response from 380 to $620 \mathrm{~nm}$. The bubbles were also recorded photographically in 20 second exposures (Figure 12). A separate photomultiplier (PMT) was used to record the light emission as a function of steam and water temperatures. No light was detected at water temperatures above $80^{\circ} \mathrm{C}-85^{\circ} \mathrm{C}$. Limited spectral measurements indicated a substantially flat spectrum in the measured range $(400-600 \mathrm{~nm})$. In spite of the high infrared sensitivity in the IIT used, no thermal emission was detected from the steam in the glass nozzle. Likewise, no infrared was detected from the collapsing steam bubbles; hence the observed luminescence cannot have been due to the steam. Instead, they determined that each collapsing bubble produced a short-duration light pulse (whose measured duration was limited by the electronics used) accompanied by a coincident sound pulse (recorded by a hydrophone) (Figure 13). Due to the nature of the source of the bubbles, the authors also used a title $V B L$ to describe this luminescence as in [3]. They also

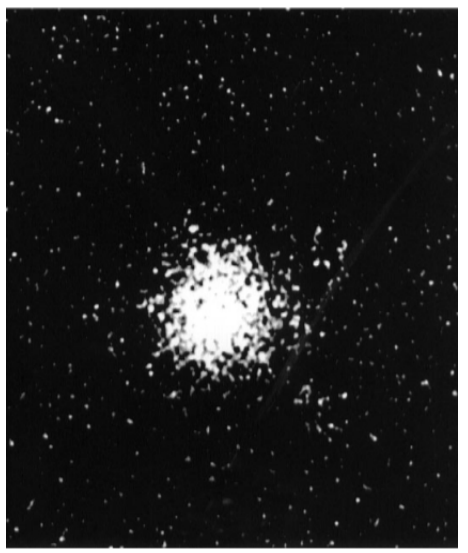

Figure 12. From [23]: $20 \mathrm{~s}$ time exposure photograph of the light produced as steam is bubbled into water at room temperature taken using the IIT-microscope system with the EHT set at $40 \mathrm{kV}$. Each spot records an individual flash.

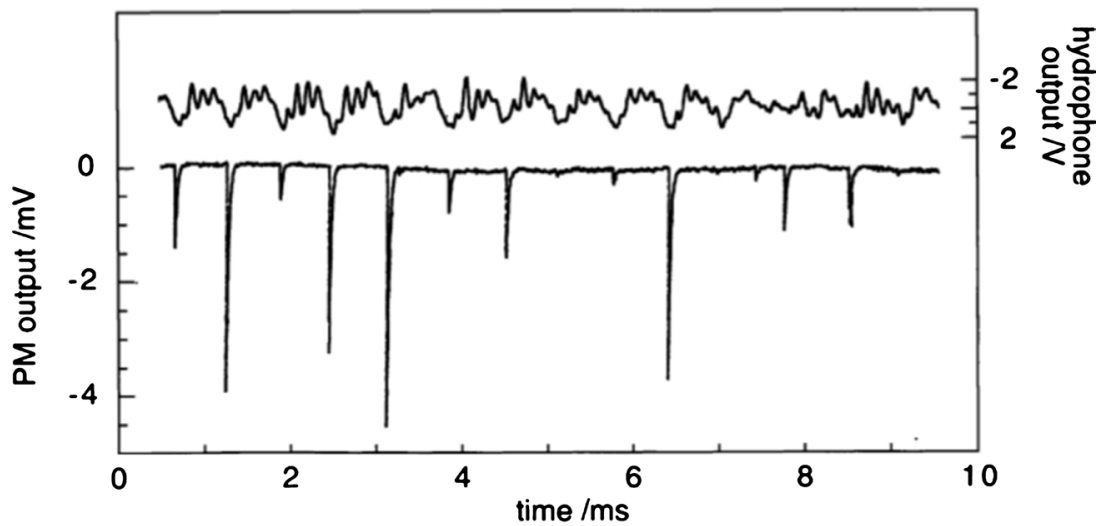

Figure 13. From [23]: The upper oscillograph trace shows the hydrophone signal as steam bubbles collapse in water; the hydrophone has a sensitivity of $30 \mathrm{mV} \cdot \mathrm{Pa}^{-1}$; the lower trace shows the light emission; each "spike" shows the light emitted by a single bubble. 
noted parallels between the $V B L$ and conventional $S B S L$.

\subsection{Detection of Artificial Bubble Luminescence Using PMT}

Reynolds later repeated the previous experiment [23] with an improved $P M T$ for light recording [24]. The $P M T$ had the advantage of superior time resolution. It was housed in a light tight cylinder, with a top insert that accommodated a 100 $\mathrm{ml}$ beaker containing room temperature water. The superheated steam was then injected into the water. As a result, clear signals were obtained from the PMT, when steam was introduced into both seawater and tap water. The difference in the response between the water types was recorded. Additionally an attempt was made to record the luminescence using an intensified $C C D$ camera, but no convincing signal was observed.

\subsection{VBL from Hydrothermal Vent Minerals and Other Hot Solids}

Reynolds then carried out additional experiments [24] to verify if vent minerals heated to $\sim 350^{\circ} \mathrm{C}$ could emit light when interacting with cold water. He used some materials from vents themselves along with other solids [25]:

1) Chips of sphalerite and chalcopyrite, from millimeter to several millimetres in scale, were mixed. The volume of material was on the order of 0.5 to $1 \mathrm{ml}$.

2) Hawaiian basalts in the form of a mixture of chips and a disk with a diameter of $25 \mathrm{~mm}$ and thickness of $1.25 \mathrm{~mm}$.

3) Samples of copper in the form of a block $(1.5 \times 2.5 \times 0.5 \mathrm{~cm})$ and iron in the form of a block $(1.5 \times 1.5 \times 0.5 \mathrm{~cm})$.

All samples were heated to a temperature of $\sim 300^{\circ} \mathrm{C}$, then were dropped into a $300 \mathrm{ml}$ beaker containing $150 \mathrm{ml}$ water at $18^{\circ} \mathrm{C}$. The beaker was placed above the $P M T$. The signal was then amplified and recorded. For all samples, a signal was observed by the $P M T$ when the hot material entered the water. Visual observations confirmed that bubbles were seen and heard as the hot material settled in the water. The bubbles were of various sizes. Thus, from all heated materials, $P M T$ records indicated flashes of light exceeding the signals previously observed in the superheated steam bubble experiments [24]. Thus, the experiments clearly demonstrated that any solids heated to a temperature of $350^{\circ} \mathrm{C}$ are capable of producing luminescence due to the formation of bubbles. The vent mineral specimens did not have any advantage over the non-vent samples. In addition to the $P M T$ experiments, the delivery system was placed in front of an intensified $C C D$ camera. As in the case of the VBL from superheated steam [24], no convincing response was observed using the $C C D$.

\section{PeTa Radiation as a Cause of Bubble Glow}

We agree that the luminescence of bubbles formed during the underwater eruption of volcanoes and geysers have the same physical nature as $C L / S L$. Model experiments [23] [24] [25] have confirmed this assumption. On the other hand, in [1] [2] [3] we have also convincingly shown that $C L, S L$, and $L I B L$ are the $P e$ - 
Ta radiation. From this, a natural conclusion that the vent luminescence and radiation recorded in model experiments are also PeTa radiation is derived. We will analyze this statement in detail, following an examination of the PeTa effect using equilibrium and non-equilibrium phase diagrams for a vapour-liquid-solid system.

\section{The PeTa Effect on a Non-Equilibrium Solid-Liquid-Vapour Phase Diagram}

The PeTa effect is the non-equilibrium radiation that accompanies first-order phase transitions. Two conditions must be met in order to obtain the PeTa effect: the transition has to occur from a less condensed phase to a more condensed one, and the transition should occur fairly quickly. Only then will a significant supersaturation of the less condensed phase be obtained, the particles (atoms, molecules, and/or clusters) of which will be in an excited state with respect to the particles of the more condensed phase, and some part of the phase transition energy will then be emitted. Several aspects of this effect have been presented in previous studies [1] [2] [3] [5] [6] [7] [26]-[45]. In this paper, we present the PeTa effect on the non-equilibrium solid-liquid-vapour phase diagram by expanding on previous work [3] and presenting additional aspects of the problem. This concept allows a clear understanding of the unified physical nature of the processes in which this effect occurs.

\subsection{Equilibrium and Non-Equilibrium Phase Diagrams}

Figure 14 and Figure 3 from [3] show equilibrium and non-equilibrium phase diagrams for vapour-liquid-solid phases. They are characterized by the presence

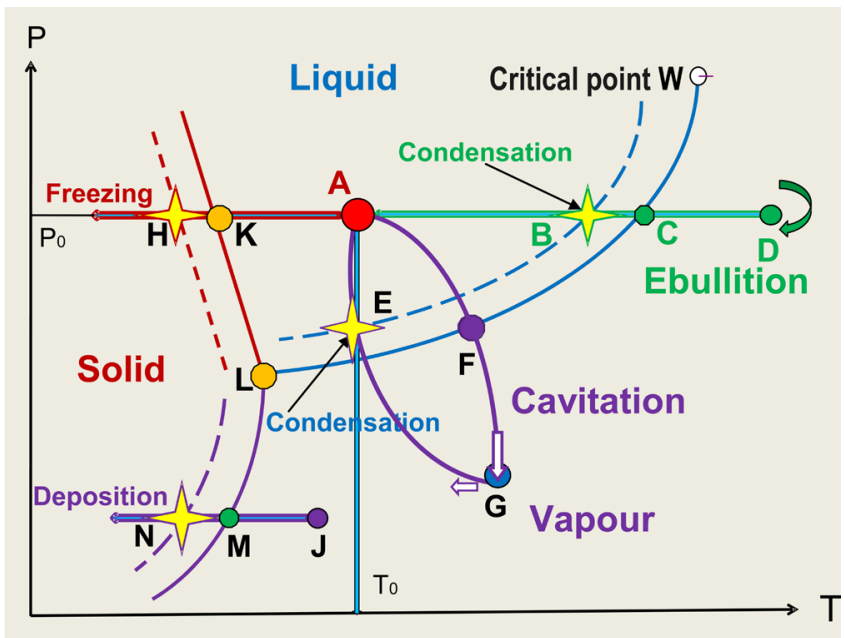

Figure 14. Presentation of the PeTa effect on nonequilibrium phase diagram for solid-liquid-vapor phases. This diagram differs from Figure 3 in [3] that in the last one the vapor-liquid transition is considered either with isobaric process (point B), or in isothermal (point E); here point B still corresponds to the isobaric process, however, point $\mathrm{E}$ corresponds to a vapor-liquid transition, when both temperature and pressure are changed at the same time. 
of non-equilibrium boundaries between the vapour-liquid, liquid-solid, and vapour-solid phases, indicated by dashed lines of the same colours. The equilibrium phase diagram for any given substance is permanent. It is constructed for idealized conditions when the rate of transition from one phase to another is infinitesimal. In contrast, the non-equilibrium phase diagram varies depending on: the direction of the phase transition (from the more condensed phase to the less condensed one or vice versa), the phase transition rate, and the phase transition conditions (amount of matter, the presence of new phase nuclei, etc.). In Figure 3 from [3] and Figure 14 schematic non-equilibrium phase diagrams are presented for transitions from less to more condensed phases: liquid-solid, vapour-liquid, and vapour-solid. For these scenarios, in comparison with equilibrium phase diagrams, the boundaries of the phase transitions are shifted toward the more condensed phases. This is an illustration of a well-known experimental fact: at high speed, the phase transition from a less condensed phase to a more condensed one (vapour-liquid, liquid-solid, or vapour-solid) occurs when the less condensed phase is supersaturated. A well-known example of this phenomenon is the supercooling of small drops of water in hail clouds to $-40^{\circ} \mathrm{C}$. This means that the water-ice transition does not occur at point $\mathrm{K}$, but instead at point $\mathrm{H}$ (Figure 3 from [3] and Figure 14), and the distance between $\mathrm{K}$ and $\mathrm{H}$ for these crystallization conditions in water is $40^{\circ} \mathrm{C}$.

\subsection{Liquid-Solid Phase Transition}

Let us consider a volume of liquid at temperature $T_{0}$ and pressure $P_{0}$ that corresponds to point A in the phase diagram (Figure 14). During the cooling of this volume of liquid at a constant pressure, the point moves from right to left. When the point reaches position $\mathrm{K}$, the liquid should freeze. However, if the cooling is rapid, freezing will occur at point $\mathrm{H}$, where the liquid will be supercooled. The distance between points $\mathrm{K}$ and $\mathrm{H}$ is larger for higher cooling rates and results in cleaner melt, smaller volume, etc. However, to meet the conditions required for observing the PeTa effect, the melt volume cannot contain less than $10^{4}-10^{5}$ crystallisable particles. In this case, some part of the phase transition energy is released in the form of radiation energy. In [1] [2] [3] [5] [6] [7] [26]-[45] and references therein there are numerous experimental observations of the PeTa radiation during crystallization of melts of various substances, including 7 alkali-halide compounds [43] [45] and sapphire [44].

\subsubsection{Conditions of the PeTa Effect in a Liquid-Solid Phase Transition}

Let us discuss the conditions under which the transition of a liquid-solid implements the PeTa effect. We have already written about this several times in our previous papers, but are forced to repeat it again and again, since the evidence of the PeTa effect does not follow from existing phase-transition conceptions, and our ideas have not yet been universally recognized. Our opponents doubt the existence of high-temperature luminescence during phase transitions and insist on the removal of the energy of the phase transition by means of thermo-conductivity, 
i.e. by phonons. Here is an example of their reasoning for a case of semiconductor melt crystallization [46]: Let us consider an excited particle near a phase-transition boundary. For phase-transition radiation to occur, the probability of excitation energy being converted into light emission by this particle at phase transition has to be equal to or greater than the probability of the excitation energy being converted to heat. But this probability is negligibly small. As an example, let us consider the crystallization from melts. For a free molecule in the excited state, its optical lifetime (the longitudinal relaxation time) $t_{1}$ is equal to $10^{-7}-10^{-8} \mathrm{~s}$. For transitions in the near-infrared range at $T \approx 1000 \mathrm{~K}$, the non-radiative multiphonon relaxation time in solids $t_{2}$ is less than or equal to $10^{-9} \mathrm{~s}$. Then, the probability of light emission is expressed by $\xi=t_{2} / t_{1} \sim 10^{-2} \ll 1$, and a radiative phase transition cannot occur.

However, in addition to our experimental and theoretical work showing that this radiation exists, there are other theoretical studies showing that, in certain cases, $\xi>1$ and radiative phase transitions are therefore possible. First order phase transitions are collective phenomena with participation of many excited particles, while previous considerations have only been made for a separate particle. For an ensemble of particles in our conditions, Dicke's observed super-radiation [47] should occur. Super-radiation occurs when a system of excited particles undergoes optical transition to a lower level due to their interactions with each other through the common radiation field, with a transition time $\left(t_{1}\right)$ that is much shorter than the radiative decay time of an individual particle. For a group of numerous particles, $t_{1}$ is a value inversely proportional to $N$, which is the number of particles participating in the phase transition. For an $N=$ $10^{5}, t_{1}$ is then equal to $10^{-12} \mathrm{~s}$, which is much less than $t_{2}=10^{-9} \mathrm{~s}$. Thus, if $N \approx$ $10^{5}$, then $\xi=t_{2} / t_{1} \sim 10^{3} \gg 1$ and a radiative phase transitions will occur. The theory of the super-radiation is well developed and has several lines of experimental evidence, but not for first-order phase transitions.

\subsubsection{PeTa Radiation under Melt Crystallization}

In almost all experiments that have detected the PeTa radiation during the crystallization of melts, substances which themselves and/or their melts are transparent to the PeTa radiation were selected: alkali-haloid compounds and lead chloride [44] [45], sapphire [31] [43], as well as a low-temperature compound, sodium thiosulphate pentahydrate (STSP) [3]. In these experiments, the PeTa radiation was recorded during the entire sufficiently long crystallisation process. However, the PeTa radiation can be detected from a supercooled melt even if the melt in the normal state is not transparent to the PeTa radiation. In [40], we called this phenomenon the transparency window for the PeTa radiation. Indeed, this system contains both the main energy level (crystal) and the exited one (supercooled melt). So, under melt-crystal phase transition this can work as an amplifier for the PeTa radiation and consequently be transparent for it. This is the only explanation for the detection of the PeTa radiation during water crystallisation, as well as in melts of $\mathrm{Te}$ and $\mathrm{Cu}$ observed experimentally. All 
these substances in both crystalline and molten states are non-transparent for the PeTa radiation. However this transparency persists for a very short time when crystallization occurs. After the PeTa radiation pulse, the energy is released, the melt crystallizes, and it is no longer transparent to the PeTa radiation. The following outlines an experiment on $T e$ crystallization that illustrates this scenario.

\subsubsection{PeTa Radiation under Te Crystallization}

A differential technique of $I R$ radiation recording has been used during crystallisation of $T e$ [48]. The layout of this setup is presented in Figure 15. The high purity tellurium $(\sim 10 \mathrm{~g})$ was sealed off under a vacuum of $0.13 \mathrm{mPa}$ in a quartz vessel (1). A similar quartz vessel was also filled with graphite powder and metal shavings (2). Both vessels were placed into the thermostat (3). The infrared radiation emitted from the vessels' bottoms were collected by mirrors (5) and lenses (6) on the thin germanium plates (7), and then by the photo-detectors (8). The differences in the electric signals from both photo-detectors were intensified by the amplifier (9) and fixed on the oscilloscope (10) or on the self-recording instrument (11). Signals from thermocouples (4) have been fixed on a self-recorder (11). In this setup, the light transmission bandwidth for the system "germanium filter-quartz" was $\lambda=2-4 \mu \mathrm{m}$. It is essential that neither melt nor crystal tellurium are not transparent in this optical range.

More than twenty experiments were carried out using this setup. The experimental results are presented in Figures 16-19. IR radiation $I$ was observed to increase sharply at moment of melt crystallisation, at point $6\left(T \sim 354^{\circ} \mathrm{C}, \Delta T \sim\right.$ $98^{\circ} \mathrm{C}$ ) (Figure 18). The radiation $I$ contains two constituents: a radiation observed only at the beginning of crystallisation (points $6-7$, Figure 18) and heat radiation. The time scanning revealed two components of the PeTa radiation (Figure 17): constant radiation with a fixed intensity of $I_{1} \approx 0.2 \times I_{m}$, and duration $0.1 \mu \mathrm{s}<t<100 \mu \mathrm{s}$; and a peak component with an intensity of $I_{2} \approx 0.5 \times I_{m}$ at $t \sim 1.5 \mathrm{~ms}$. The beginning of the second component was observed at $t \sim 1 \mathrm{~ms}$, with a total duration of $\sim 200 \mathrm{~ms}$. Then it was observed increasing the $I$ (point 8 , Figure 17) most likely due to increased temperature of the supercooled melt at

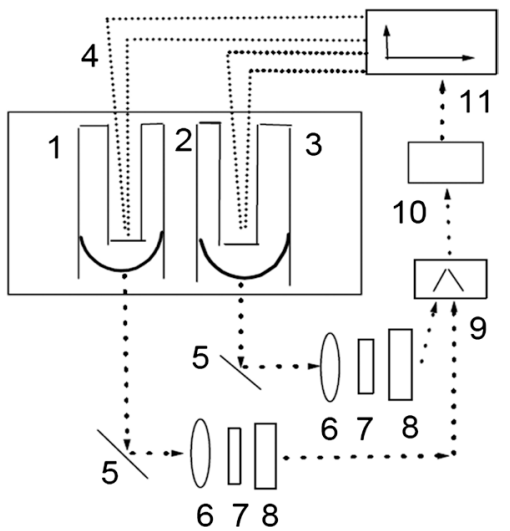

Figure 15. From [48]: The layout drawing of the installation for Te crystallization. 


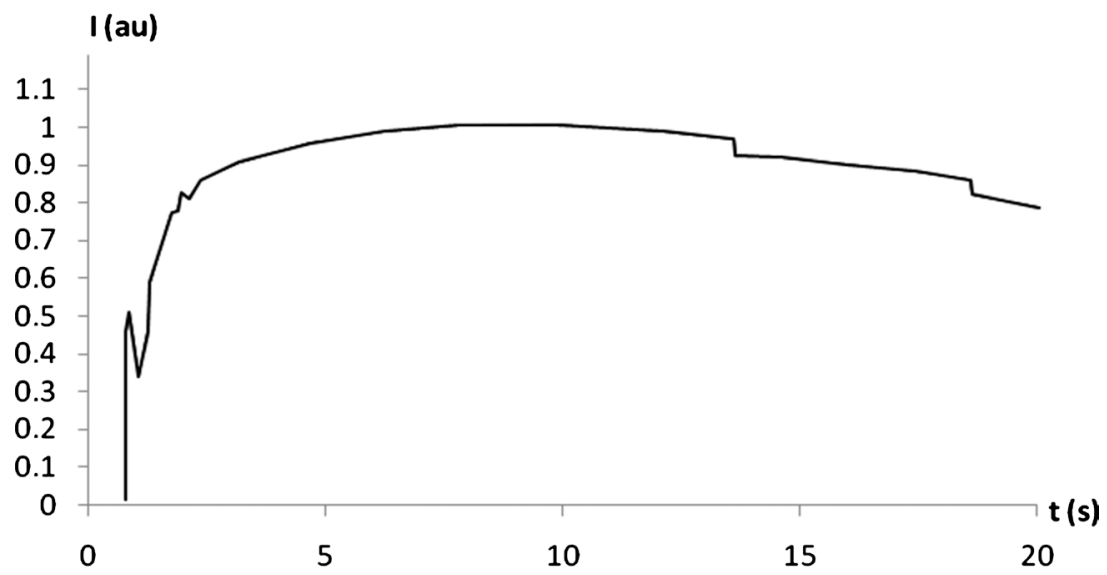

Figure 16. From [48]: The time-dependence of radiation intensity $I(t)$ ( $I$-arbitrary units, $t$-sec) at tellurium melt crystallization.

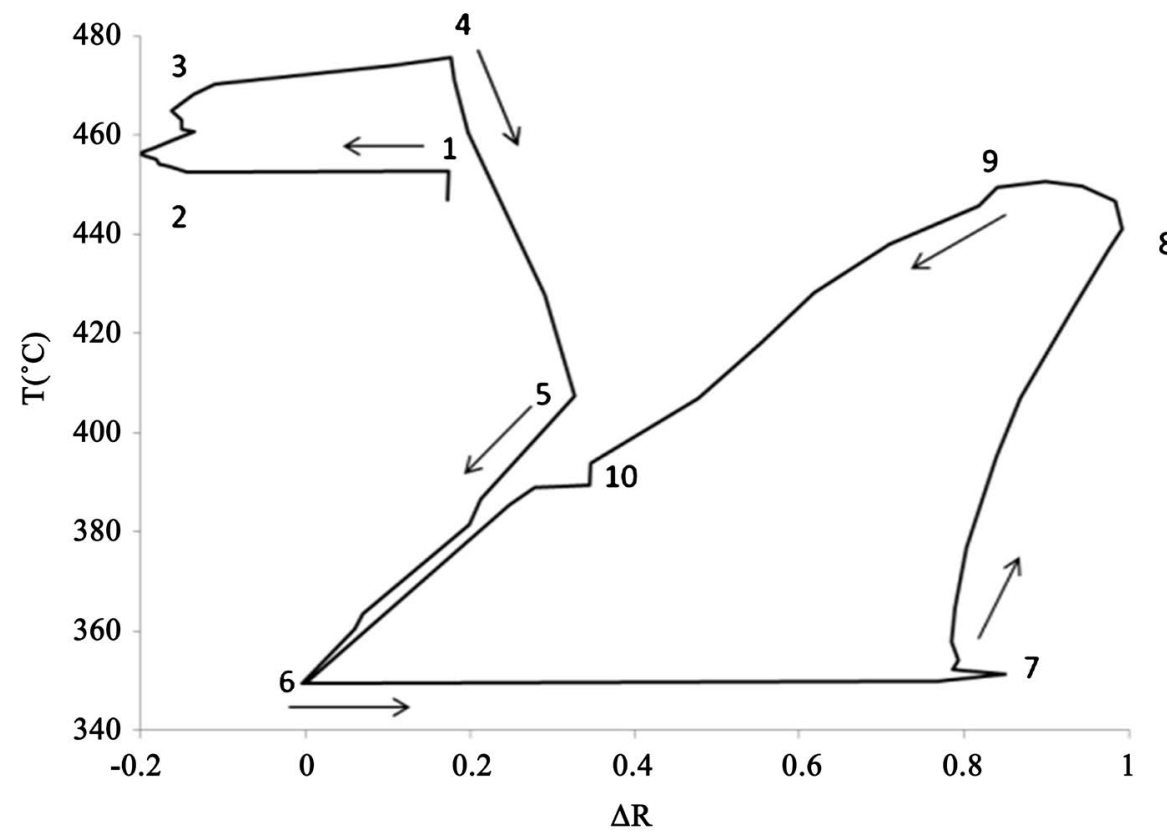

Figure 17. From [48]: The temperature dependence of difference of radiation intensity $\Delta R$ at one cycle of the tellurium melting-crystallization.

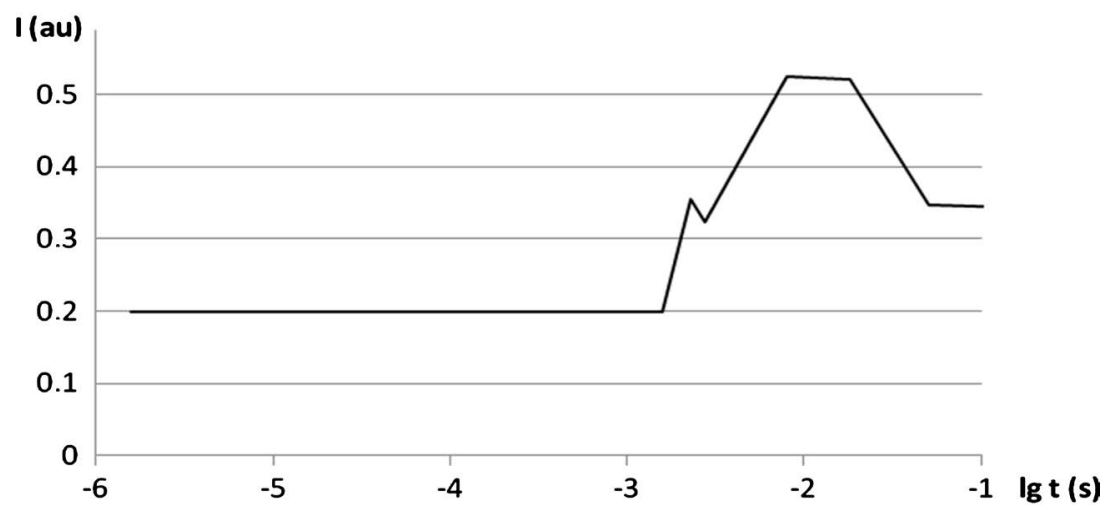

Figure 18. From [48]: The logarithmic time-dependence of characteristic radiation intensity $I(\lg t)(I$-arbitrary units, $t$-sec) at the initial stage. 


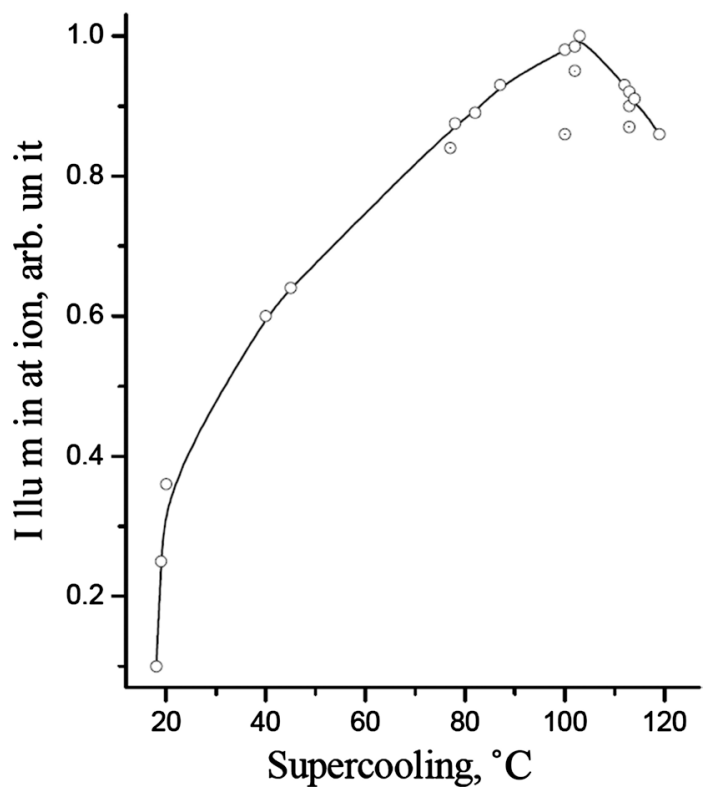

Figure 19. From [48]: The dependence of intensity of characteristic radiation $I_{2}$ from melt super-cooling $\Delta T$.

the beginning of the crystallisation process. Two small and sharp decreases in $I$ occurred at crystallization completion (points 9 - 10, Figure 17), which probably were caused by the changing radiation coefficient of the solid tellurium-quartz.

The full cycle of melt-crystallization is presented in Figure 18 for the dependence $\Delta R-T$, where $\Delta R$ is a differential intensity of radiation. Here points $1-2$ designate the "melting shelf" with a decrease in $\Delta R$ at a constant $T$. The radiation coefficient of liquid tellurium is less than the coefficient of solid tellurium. During melt overheating $\Delta T \approx 20^{\circ} \mathrm{C}$ (points 2-3-4), the $\Delta R$ changed in a sawtooth pattern, then increased again. During further melt cooling, the $\Delta R$ increased and decreased with a noticeable break at $\sim 410^{\circ} \mathrm{C}$ (points $4-5-6$ ). Namely the temperature radiation characteristics of the overheating and supercooled melt are not similar at points 2 - 6 (thermal memory phenomenon). At the nucleation moment $\left(\sim 354^{\circ} \mathrm{C}\right)$, a sharp peak of PeTa radiation was observed (points $6-7$ ) and $\Delta R$ again increased to a maximum (point 8), near the temperature maximum (points 8 - 9). During the cooling of solid tellurium, the two small anomalies ("small shelves") reveal $\Delta R\left(T \sim 390^{\circ} \mathrm{C}\right.$ and $420^{\circ} \mathrm{C}$, points $\left.10-11\right)$. In addition, if the super-cooling of the melt had been less than $\Delta T \sim 18^{\circ} \mathrm{C}$, the peak of $P e T a$ radiation was not observed (Figure 18), rather, the PeTa effect for Te has a clear threshold for occurrence. We observed the same threshold effect in all our experiments during crystallisation of the other investigated substances. A maximum of the $\mathrm{PeTa}$ radiation intensity occurred at $\Delta T \approx 106^{\circ} \mathrm{C}-108^{\circ} \mathrm{C}$ (Figure 18), which corresponds to the maximum rate of nucleation in the tellurium melt [49].

Recording $P e T a$ radiation in this $I R$ range provides support for our window of transparency ( $W T$ ) model for PeTa radiation in supercooled melts [40]. It allows also the design of a cavity-free pulsed laser on the basis of supercooled melt 
crystallisation [39]. The short duration of PeTa radiation shows that the $W T$ is closed when there are many nucleated seeds in the melt. The latent energy of $T e$ melt crystallization is $17.5 \mathrm{~kJ} / \mathrm{mol}$. With respect to formula (2) from [2], the recorded radiation corresponds to $\lambda_{1}^{(C, 2)} \sim 3.4 \mu \mathrm{m}$, which is the second harmonic of the base radiation. Unfortunately, the experimental system did not allow us to investigate if other harmonics, especially the base radiation $\lambda_{1}^{(C, 1)}=6.8 \mu \mathrm{m}$, were present. However, with respect to our model, these results can be interpreted as the formation of the dimers of $T e$ atoms in the melt before crystallisation. In our calculations, we did not take into account the binding energy of $T e$ atoms during the formation of a dimer since we did not find these data in the literature.

\subsubsection{Phase Transition Energy during Melt Crystallization Emitted in the Form of the PeTa Radiation}

The problem of the PeTa radiation yield during melt crystallization has not been solved. It is evident that the yield depends on the transparency of both phases. In previous studies based on experimental data, we estimated the yield of the PeTa radiation for the following substances: alkali-halide compounds [44] [45], sapphire [31] [43], and STSP [3]. For $S T S P$ the yield was $\sim 1 \%$, for $K B r$ it was a few percent, and for sapphire the yield was up to $30 \%$. Sapphire yielded interesting results, as a sapphire crystal is much more transparent to the PeTa radiation than its melt. This allowed us to use a filter system to make a film of layer-by-layer crystallised, supercooled sapphire melt [31]. PeTa radiation always propagated in the direction of crystal growth. In particular, during the layer-by-layer growth of the crystal, the PeTa radiation propagated parallel to the surface of the melt.

\subsubsection{Estimation of the Te Drop Solidification Time}

We estimated time $\tau_{\mathrm{s}}$ of the Te drop solidification when cooling through the Plank radiation effect without PeTa radiation. For this, it is feasible to use the solution of Stephan's problem under the following assumptions: PeTa radiation is absent, the shape of the liquid drop is a sphere of diameter $2 R_{0}$, freezing starts at the center of the drop, the constant solidification temperature $T=T^{*}$ in $\mathrm{K}$ is applied on a movable boundary of the solidification front, the heat of crystallisation emanates from the movable boundary, the heat from the external surface is removed by radiance emission, $T_{0}$ and $T_{\mathrm{E}}$ are temperatures of the supercooled melt on the external surface of the drop and in the environment, respectively, in K. The formulation of the problem in the integral form of thermo conductivity equations for both crystallized and liquid volumes allows the following simple formula to be derived:

$$
\tau_{s}=\left[\Lambda^{m v}-C_{L}\left(T^{*}-T_{0}\right)\right] R_{0} / 3 \varepsilon_{s} \sigma\left(T_{0}^{4}-T_{E}^{4}\right)
$$

where $\varepsilon_{s}$ is the averaged coefficient of emission from the surface of the melt; $C_{L}$ is the specific heat of the melt; $\Lambda^{m v}$ is the latent melting heat for a unit of volume; and $\sigma$ is the Stephan-Boltzmann constant. The progression of the so- 
lidification front inside the drop is controlled by the surface cooling mechanism.

For the experiment with $T e$ :

Mass $m=10 \mathrm{~g}$ and density of the melt $\rho_{L}=5.7 \mathrm{~g} \cdot \mathrm{cm}^{-3}$, thus, $R_{0}=7.5 \times 10^{-3} \mathrm{~m}$, $T^{*}=723 \mathrm{~K}$, supercooling is $98 \mathrm{~K}$, that is $T_{0}=625 \mathrm{~K} ; T_{E}=293 \mathrm{~K}$;

$C_{L}=1.148 \times 10^{6} \mathrm{~J} \cdot \mathrm{m}^{-3} \cdot \mathrm{K}^{-1}$; latent melting heat is $\Lambda_{m}=17.5 \mathrm{~kJ} \cdot \mathrm{mol}^{-1}$, that is $\Lambda^{m v}=7.855 \times 10^{8} \mathrm{~J} \cdot \mathrm{m}^{-3}$. We obtained $\tau_{\mathrm{s}}=\left(206 / \varepsilon_{\mathrm{s}}\right) \mathrm{s}$. The coefficient of emission $\varepsilon_{s}$ has to be chosen from the range $0.1-0.95$. We chose $\varepsilon_{s}=0.95$, keeping in mind that our last assumption concerning constancy of the temperature $T_{0}$ on the surface of the drop may not be exactly correct. In this case, $\tau_{\mathrm{s}}=217 \mathrm{~s}$, but we did not take into account the heat exchange on the surface of the quartz drop. Heat exchange affected by free convection is comparable with the radiance emission at a surface temperature of $\sim 1250 \mathrm{~K}$ [42]. In our case, the surface temperature is two times less. Thus, at a temperature of $650^{\circ} \mathrm{C}$, the heat exchange by conductive mechanism can be estimated as approximately 16 times more efficient than exchange by radiance emission (due to $T_{S}^{4}$ functional dependence of the latter) and we obtain $\tau_{s} \sim 14 \mathrm{~s}$. In the experiment, the time of solidification was $\sim 20 \mathrm{~s}$. This means that, for $T e$ crystallisation, only a small part of the latent heat (order of accuracy of our estimate) is radiated through the PeTa radiation at the very beginning of nucleation.

There are still many interesting questions in this area of study that need to be addressed: How does crystallisation occur in the presence or absence of a massive seed? How is a critical volume created at the crystallization front and in a free volume? Is there a correlation between the critical volume and the value of the critical nucleus in classical theory? Is this phenomenon possible in sapphire or in alkali-halide compounds that with $T e$ ?

\subsection{PeTa Effect in the Vapour-Solid Transition}

Let us consider a volume of vapour that corresponds to point $J$ on the phase diagram (Figure 3 from [3] and Figure 14). The cooling of this volume of vapour corresponds to a horizontal movement of the point from right to left. When the point reaches position $\mathrm{M}$, the vapour should crystallise. If the cooling is rapid, crystallisation will occur at point $\mathrm{N}$, where the vapour will be supercooled. In this case, some part of the phase transition energy is released in the form of the $P e T a$ radiation. We investigated this radiation in detail during the deposition of water vapour and other components of air on ice at temperatures of $77-253 \mathrm{~K}$ [2] [9] [10] [11] [12].

\subsection{PeTa Effect in the Vapour-Liquid Phase Transition}

Let us consider a volume of vapour that corresponds to point $\mathrm{D}$ on the phase diagram (Figure 3 from [3] and Figure 14). The cooling of this volume of vapour corresponds to a horizontal movement of the point from right to left. When the point reaches position $\mathrm{C}$, the vapour should condense. If the cooling is rapid, condensation will occur at point $\mathrm{B}$, where the vapour will be supercooled. 
In this case, a significant part of the phase transition energy is released in the form of the PeTa radiation. The most impressive illustration of this phenomenon is infrared radiation in the atmospheric transparency window of $7-14 \mu \mathrm{m}$ during the formation of clouds [2] [29].

\subsection{Vapour Bubble Luminescence ( $V B L)$}

It is this mechanism that functions in our model of bubble glow at hydrothermal vents. A description of the $V B L$ phenomenon is as follows. For subsequent sections, we have considered a very small volume of liquid at temperature $T_{0}$ and pressure $P_{0}$ that corresponds to point A on the phase diagram (Figure 3 from [3] and Figure 14).

\subsubsection{Temperature Change (LIBL Illustration)}

Under the action of a local heat source on this small volume, the temperature rises sharply. Such heat sources may include: a concentrated laser beam which leads to an $L I B L$, electric discharge arc, or some other source of heat such as a heated solid placed in water. As the temperature rises, the thermodynamic state of the volume under consideration will then correspond to the horizontal movement of the point on the phase diagram to the right of position A (Figure 3 from [3] and Figure 14). When the point reaches position C, one or several vapour bubbles should appear in this volume of liquid, corresponding to boiling of the liquid. The appearance of bubbles in a boiling liquid is a rather complex physical process. In particular, this is due to pressure. The horizontal motion of the point corresponds to an isobaric process. In our previous papers [2] and [3], we showed that this approach is applicable to $L I B L$ in water and liquid gases when the diameters of the bubbles are on the order of a millimetre. However, when a bubble nucleates its size is very small and the Laplace pressure inside the bubble may be very large. In this case, the isobaric approximation is not applicable. However, we ignore the process of nucleation, because only the further behaviour of a large volume bubble is essential in this situation. The position of point $\mathrm{D}$ on the phase diagram corresponds to the condition when the energy source is exhausted and the bubble has reached its maximum size. Then, under the action of the heat lost to the external environment, the bubble begins to cool. It is very important that the point on the phase diagram changes its direction of movement at this time. For a short time, its speed of movement is zero. Therefore, the thermodynamic conditions inside the bubble at this time will be in equilibrium. This is a critical assumption, as it allows us to determine the thermodynamic characteristics of the vapour inside the bubble. In our previous papers [2] and [3] on the study of $L I B L$ in water or liquid gases, this corresponds to a $\sim 1 \mathrm{~mm}$ bubble size and a vapour temperature of $\sim 120^{\circ} \mathrm{C}$ for water vapour, or $\sim 96 \mathrm{~K}$ for a bubble inside liquid nitrogen. In water, in addition to the vapour, there is a small amount of gas inside the bubble that was previously dissolved. For the spectrum of the subsequent luminescence, it is very important that several gases contribute to the formation of clusters in the vapour. 
At this stage, if the cooling rate is high enough, the behaviour of the vapour resembles the behaviour of an ideal gas. Its equilibrium pressure decreases, and under the influence of external pressure the bubble size decreases. This isobaric process corresponds to the movement of the point on the phase diagram to the left from point $\mathrm{D}$ to point $\mathrm{C}$. For such behaviour to occur, a very high cooling speed is required, at which the rate of change of the linear dimensions of the bubble must be at least $0.5 \mathrm{~m} / \mathrm{s}$. As previously shown [1] [2] [3], under this condition the vapour will not have time to condense on the inner surface of the bubble at point $\mathrm{C}$. When the point passes to the left of point $\mathrm{C}$ and is in the equilibrium zone for existence of a liquid, the substance inside the bubble will not be a liquid, but instead is compressed supercooled vapour. At elevated pressures, in supersaturated vapour approaching point $B$, eight processes consistently occur: 1) The vapour molecules are pre-disposed to form clusters, and this most likely occurs; 2) Molecules/clusters become excited compared to the bulk liquid molecules, because the equilibrium state of matter here is liquid; 3) The density of molecules/clusters in the vapour increases; 4) In conformity with Dicke [30], at point $B$ the threshold value of this density is reached and there is an interaction between excited molecules and clusters through the collective radiation field; 5) At point $\mathrm{B}$ we obtain the classical situation in which the PeTa radiation occurs, and at this moment there is an instant condensation of the vapour inside the bubble, with the emission of phase-transition energy as the PeTa radiation (resulting in a flash); 6) There is an instantaneous pressure drop; 7) There is a collapse of the bubble as a result of the pressure drop; 8) There is subsequent formation of a shock wave.

Another option is that, in some device (for example, a cappuccino machine) there is a boiling liquid with intense bubble formation. Then, these bubbles are introduced into a large volume of a cold liquid. Suppose that the physico-chemical state of the vapour inside these bubbles corresponds to point $\mathrm{D}$ on the phase diagram. In cold water, the bubble begins to cool rapidly, which corresponds to the movement of the point from position $\mathrm{D}$ to position $\mathrm{B}$. This is fully consistent with the process described above. Upon reaching position $\mathrm{B}$, there will be the $P e T a$ flash and a collapse of the vapour bubble. So, in principle, we can observe $V B L$ every time we make a cappuccino.

\subsubsection{Pressure Change ( $C L / S B S L / M B S L$ Illustration)}

Under the action of a stretching mechanical wave propagating in a liquid, the pressure in the small volume around point A can decrease rapidly. This mechanical wave could be, for example, an ultrasonic wave, which leads to the SL effect. At constant temperature, the thermodynamic state of the volume under consideration will then correspond to the vertical downward movement of the point on the phase diagram from position A to position F (Figure 3 from [3]). When the point reaches position $\mathrm{F}$, vapour bubbles should appear in this volume of the liquid. The further behaviour of the bubbles corresponds to the vertical motion of the point and repeats the conditions of previous case if we replace a 
rise and fall of temperature, respectively, with a decrease and increase in pressure. The position of point $\mathrm{G}$ will then be equivalent to the position of $\mathrm{D}$, of point $\mathrm{F}$ to point $\mathrm{C}$, and of point $\mathrm{E}$ to point $\mathrm{B}$. Thus, a flash with the release of condensation energy as the PeTa radiation will occur at point $\mathrm{E}$.

\subsubsection{Both Pressure and Temperature Change Simultaneously}

It is clear from the phase diagram (Figure 14) that the processes of $C L / S B S L / M B S L$ need not occur in isothermal conditions, nor does the process of $V B L$ require isobaric conditions. It is possible to imagine a situation where simultaneous changes in temperature and pressure take place in the volume under consideration. It is this situation that is realized with bubble glow at hydrothermal vents, when water heated to $400^{\circ} \mathrm{C}$, which is under high pressure, leaves the vent into cold water. This case will correspond to a more complex trajectory of the movement of the point on the phase diagram. However, observing the phenomenon of the PeTa radiation requires movement of the point that must correspond to the following conditions:

1) When a point moves in both forward and reverse directions, the trajectory must cross the liquid-vapour phase boundary between the triple $\mathrm{L}$ and critical $\mathrm{W}$ points.

2) The speed of the reverse movement of the point on the phase diagram must be high enough to eliminate the equilibrium condensation of vapour on the walls of the bubble.

3) When describing the $S B S L$ process, the trajectory of the point on the phase diagram should be repeated with each cycle: i.e. return to position A, since the process is repeated millions of times without a noticeable change in physical conditions.

4) Possible trajectories of the point on the phase diagram provide the phase diagrams for real liquids and the limits of the values in which temperature and pressure can be changed under $V B L$.

5) These findings are very useful in further applications and for improving our $V B L$ model.

\section{Conclusions}

Here is a list of the main events that led to the discovery and explanation of the nature of the glow in deep-sea vents:

1) Plate tectonic theory had predicted the existence of hydrothermal vents, which are deep-sea hot springs. Their existence was confirmed in 1977.

2) In 1989, researchers observed that the Rimicaris exoculata deep-sea shrimp living in vent areas have photoreceptors similar to those of humans and are sensitive to daylight. The presence of these photoreceptors indicates that sources of this kind of light must be near the shrimp. It turned out that the sources are the high-temperature hydrothermal vents themselves.

3) Spectra of hydrothermal vents were studied using deep-water spectrometers OPUS and ALISS. A significant part of the radiation turned out to be con- 
centrated in the visible range of the spectrum. It has been associated with the presence of bubbles, since the radiation intensity correlated with the number of bubbles. Model experiments, especially with a cappuccino machine, confirmed these assumptions. However, the physical nature of the radiation remained incomprehensible.

4) The PeTa model for bubble glow formed during seafloor hydrothermal activity convincingly explains the results of field observations and modeling experiments. The essence of the model is as follows: Water heated to $400^{\circ} \mathrm{C}$ and under high pressure breaks out of the vent into cold $\left(2^{\circ} \mathrm{C}\right)$ ocean water. When pressure drops, numerous hot steam bubbles form in the water (an analogous flow scenario in a Venturi tube). With rapid cooling of steam bubbles in cold water, the steam inside them instantly condenses with the emission of condensation energy in the form of PeTa radiation.

5) This model is only part of the manifestation of the PeTa radiation in nature, shown in the nonequilibrium phase diagram (Figure 14) and described in papers [1]-[7] [26]-[45].

\section{Conflicts of Interest}

The author declares no conflicts of interest regarding the publication of this paper.

\section{References}

[1] Tatartchenko, V.A. (2017) Sonoluminescence as the PeTa Radiation. Optics and Photonics Journal, 7, 27-55. https://doi.org/10.4236/opj.2017.72004

[2] Tatartchenko, V.A. (2017) Sonoluminescence as the PeTa Radiation, Part Two. Optics and Photonics Journal, 7, 197-220. https://doi.org/10.4236/opj.2017.711019

[3] Tatartchenko, V.A. (2018). Sonoluminescence as the PeTa Radiation, Part Three. Optics and Photonics Journal, 8, 187-200. https://doi.org/10.4236/opj.2018.86017

[4] Ravilous, K. (2010) Cloud Power. New Scientist, 208, 38-41. https://doi.org/10.1016/S0262-4079(10)62951-X

[5] Perel'man, M.E. and Tatartchenko, V.A. (2007) Phase Transitions of the First Kind as Radiation Processes. 1-17. https://arxiv.org/ftp/arxiv/papers/0711/0711.3570.pdf

[6] Perel'man, M.E. and Tatartchenko, V.A. (2008) Phase Transitions of the First Kind as Radiation Processes. Physics Letters A, 372, 2480-2483. https://doi.org/10.1016/j.physleta.2007.11.056

[7] Tatartchenko, V.A. (2009) Some Peculiarities of First Order Phase Transitions. Reviews on Advanced Materials Science, 20, 58-69.

[8] Ayad, M. (1971) Electromagnetic Radiation during Ultrasonic Cavitation. Infrared Physics, 11, 249. https://doi.org/10.1016/0020-0891(71)90027-3

[9] Cristina, L. (2012) Life on the Ocean Floor, 1977; the Discovery of Deep-Sea Hydrothermal Vents along the Galápagos Rift Revealed a Biological Garden of Eden. The Scientist, Sep. 1, 2012. https://www.the-scientist.com/foundations/life-on-the-ocean-floor-1977-40523

[10] Van Dover, C.L., Szuts, E.Z., Chamberlain, S.C. and Cann, J.R. (1989) A Novel Eye in "Eyeless" Shrimp from Hydrothermal Vents of the Mid-Atlantic Ridge. Nature, 
337, 458-460. https://doi.org/10.1038/337458a0

[11] Smith, M.O. and Delaney, J.R. (1989) Variability of Emitted Radiation from Two Hydrothermal Vents. Eos, Transactions American Geophysical Union, 70, 1161. https://doi.org/10.1029/89EO00390

[12] Tivey, M.K. (1995) Modeling Chimney Growth and Associated Fluid Flow at Seafloor Hydrothelmal Vent Sites. In: Humphris, S.E., Zierenberg, R.A., Mullineaux, L.S. and Thomson, R.E., Eds., Seafloor Hydrothermal Systems. Physical, Chemical, Biological, and Geological Interactions, AGU, Washington DC, 158-177.

[13] White, S.N. (2000) An Investigation into the Characteristics and Sources of Light Emission at Deep-Sea Hydrothermal Vents. Thesis for the Degree of Doctor of Philosophy at the Massachusetts Institute of Technology and the Woods Hole Oceanographic Institution. https://doi.org/10.1575/1912/4053

[14] Saet, A.I. and Tatarchenko, V.A. (1970) Density Stratification in a Liquid during Temperature Changes. Journal of Applied Mechanics and Technical Physics, 11, 336-337. https://doi.org/10.1007/BF00908118

[15] Saet, A.I. and Tatarchenko, V.A. (1973) Connection between Convective Instability in a Melt and Stratified Distribution of Impurities during Crystallization. Bulletin of the Academy of Sciences of the USSR, Physical Series, 36, 541-542.

[16] Van Dover, C.L., Cann, J.R., Cavanaugh, C., Chamberlain, S.C., Delaney, J.R., Janecky, D., Imhoff, J., Tyson, J.A. and Participants, L.W. (1994) Light at Deep Sea Hydrothermal Vents. EOS, 75, 44-45. https://doi.org/10.1029/94EO00556

[17] Van Dover, C.L., Reynolds, G.T., Chave, A.D. and Tyson, J.A. (1996) Light at Deep-Sea Hydrothermal Vents. Geophysical Research Letters, 23, 2049-2052. https://doi.org/10.1029/96GL02151

[18] White, S.N., Bailey, J.W., Van Dover, C.L. and Chave, A.D. (1996) Measurements of Light at Hydrothermal Vents. RIDGE Events, 7, 10-12.

[19] White, S.N., Chave, A.D., Bailey, J.W., Van Dover, C.L. and Reynolds, G.T. (1996) Measurements of Light at Hydrothermal Vents, $9^{\circ} \mathrm{N}$ East Pacific Rise. Eos, Transactions American Geophysical Union, 77, F404.

[20] White, S.N., Chave, A.D., Reynolds, G.T., Gaidos, E.J., Tyson, J.A. and Van Dover, C.L. (2000) Variations in Ambient Light Emission from Black Smokers and Flange Pools on the Juan de Fuca Ridge. Geophysical Research Letters, 27, 1151-1154. https://doi.org/10.1029/1999GL011074

[21] White, S.N., Chave, A.D., Reynolds, G.T. and Van Dover Cindy, L. (2002) Ambient Light Emission from Hydrothermal Vents on the Mid Atlantic Ridge. Geophysical Research Letters, 29, 34-1-34-4. https://doi.org/10.1029/2002GL014977

[22] White, S.N., Alan, D., George, C. and Reynolds, T. (2002) Investigations of Ambient Light Emission in Deep-Sea Hydrothermal Vents. Journal of Geophysical Research, 107, EPM 1-1-EPM 1-13. https://doi.org/10.1029/2000JB000015

[23] Avik, C. and Walton, A.J. (2001) Light Emission from Collapsing Superheated Steam Bubbles in Water. Journal of Luminescence, 92, 27-33. https://doi.org/10.1016/S0022-2313(00)00250-7

[24] Reynolds, G.T. (2000) Evidence for Vapor Bubble Luminescence. Technical Report \#1. Princeton University, Joseph Henry Laboratories, Department of Physics, Princeton, ONR Grant N00014-00-1-0010.

[25] Reynolds, G.T. (2001) Vapor Bubble Luminescence from Deep Sea Hydrothermal Vent Minerals and Other Hot Solids. Technical Report \#5. Princeton University, Joseph Henry Laboratories, Department of Physics, Princeton, ONR Grant N00014-00-1-0010. 
https://doi.org/10.21236/ADA389585

[26] Tatartchenko, V.A., Smirnov, P.V. and Wu, Y. (2014) PeTa Radiation under Ice Deposition. 13th International Conference on the Physics and Chemistry of Ice, Hanover, 17-20 March 2014, Abstracts, Poster A19.

[27] Tatartchenko, V.A., Smirnov, P.V. and Wu, Y. (2013) First Order Phase Transitions as Radiation Processes. Optics and Photonics Journal, 3, 1-12. https://doi.org/10.4236/opj.2013.38A001

[28] Tatartchenko, V.A., Smirnov, P.V. and Jin, H.R. (2014) First Order Phase Transitions as Radiation Processes, Part Two. Optics and Photonics Journal, 4, 26-37. https://doi.org/10.4236/opj.2014.42005

[29] Tatartchenko, V.A. (2013) Characteristic IR Radiation under Crystallization, Sublimation, and Condensation (PeTa Effect). Scientific Program and Book of Abstracts, 17 th ICCGE, Warsaw, 34.

[30] Vitali, T., Liu, Y.-F., Wu, Y., Zhou, J.-J., Sun, D.-W., Yuan, J., Zhu, Z.-Y., Pavel, S., Artem, R., Niu, S.-J., Li, D.-Z., Zong, Z.-Y. and Chen, X.-F. (2013) Infrared Characteristic Radiation under First Order Phase Transitions-Melt Crystallization and Vapor Condensation or Deposition. Acta Physica Sinica, 62, Article ID: 079203.

[31] Tatartchenko, V.A., Liu, Y.F., Chen, W.Y., Pavel, S. and Sun, D.W. (2012) Characteristic IR Radiation during Crystallization. Proceedings of the 7 th IWMCG, Taipei, 26.

[32] Tatartchenko, V.A. (2010) Infrared Characteristic Radiation of Water Condensation and Freezing in Connection with Atmospheric Phenomena. Earth Science Reviews, 101, 24-28. https://doi.org/10.1016/j.earscirev.2010.03.002

[33] Tatartchenko, V.A. (2011) Infrared Characteristic Radiation of Water Condensation and Freezing in Connection with Atmospheric Phenomena; Part 2: New Data. Earth Science Reviews, 107, 311-314. https://doi.org/10.1016/j.earscirev.2011.04.001

[34] Tatartchenko, V.A., Liu, Y.F., Chen, W.Y. and Smirnov, P.V. (2012) Infrared Characteristic Radiation of Water Condensation and Freezing in Connection with Atmospheric Phenomena; Part 3: Experimental Data. Earth Science Reviews, 114, 218-223. https://doi.org/10.1016/j.earscirev.2012.07.001

[35] Tatartchenko, V.A, Liu, Y.F., Chen, W.Y., Zhou, J.J., Zhu, Z.Y., Smirnov, P.V., Niu, S.J., Li, D.Z. and Lu, Y.F. (2012) Cloud Power. Lecture Notes in Information Technology, Vol. 9, 191-196.

[36] Tatartchenko, V.A. (2010) Infrared Characteristic Radiation of First Order Phase Transitions in Connection with Optics of Atmosphere. Atmospheric and Oceanic Optics, 23, 169-175. https://doi.org/10.1134/S1024856010040020

[37] Tatartchenko, V.A. (2010) Nature of Some Sources of Atmospheric Infrared Radiation. Investigation of Earth from Space, No. 2, 88-90. (In Russian)

[38] Tatartchenko, V.A. (2010) The Nature of Specific Sources of Infrared Radiation Recorded by Satellites. Modern Problems of Distance Probes of Earth from Space, Institute of Space Research of Russian Academy of Sciences, Moscow, Vol. 7, No. 4, 310-318. (In Russian)

[39] Tatartchenko, V.A. (2009) Infrared Laser Based on the Principle of Melt Crystallization or Vapor Condensation. Why Not? Optics \& Laser Technology, 41, 949-952. https://doi.org/10.1016/j.optlastec.2009.04.001

[40] Tatartchenko, V.A. (2008) Characteristic IR Radiation Accompanying Crystallization and Window of Transparency for It. Journal of Crystal Growth, 310, 525-529. https://doi.org/10.1016/j.jcrysgro.2007.11.155 
[41] Perel'man, M.E., Rubinstein, G.M. and Tatartchenko, V.A. (2008) Mechanisms of Dendrites Occurrence during Crystallization: Features of the Ice Crystals Formation. Physics Letters, 372, 4100-4103. https://doi.org/10.1016/j.physleta.2008.03.009

[42] Tatarchenko, V.A. (1993) Shaped Crystal Growth. Kluwer, London. https://doi.org/10.1007/978-94-017-2988-8

[43] Umarov, L.M. and Tatarchenko, V.A. (1984) Differential Spectra of Crystallization Radiation of Alkali-Metal Halides. Soviet Physics, Crystallography, 29, 670-673.

[44] Tatarchenko, V.A. and Umarov, L.M. (1980) Infrared Radiation Accompanying the Crystallization of Sapphire. Soviet Physics, Crystallography, 25, 748-749.

[45] Tatarchenko, V.A. (1979) Appearance of Distinguishing Features in Emission Spectra during Crystallization of Substances Transparent in the IR Region. Soviet Physics, Crystallography, 24, 238-239.

[46] Sall, S.A. and Smirnov, A.P. (2000) Phase Transition Radiation and the Growth of a New Phase. Technical Physics, 45, 849-853. https://doi.org/10.1134/1.1259737

[47] Dicke, R.H. (1954) Coherence in Spontaneous Radiation Processes. Physical Review, 93, 99-110. https://doi.org/10.1103/PhysRev.93.99

[48] Makukha, K., Kidyarov, B.I., Nikolaev, I.V. and Kozharo, A.P. (2005) Proceedings of KORUS-2005, the 9th Russian-Korean International Symposium on Science and Technology. Institute of Electrical \& Electronics Engineers, Piscataway, 222-224.

[49] Nikolaev, I.V. and Kidyarov, B.I. (1977) Installation for Statistic Thermal Analysis. Industrial Laboratory, 43, 564-567. (In Russian) 